\title{
Experimental Review of Neural-based approaches for Network Intrusion Management
}

\author{
Mario Di Mauro, Member, IEEE, Giovanni Galatro, Antonio Liotta, Senior Member, IEEE
}

\begin{abstract}
The use of Machine Learning (ML) techniques in Intrusion Detection Systems (IDS) has taken a prominent role in the network security management field, due to the substantial number of sophisticated attacks that often pass undetected through classic IDSs. These are typically aimed at recognizing attacks based on a specific signature, or at detecting anomalous events. However, deterministic, rule-based methods often fail to differentiate particular (rarer) network conditions (as in peak traffic during specific network situations) from actual cyber attacks. In this paper we provide an experimental-based review of neural-based methods applied to intrusion detection issues. Specifically, we $i$ ) offer a complete view of the most prominent neural-based techniques relevant to intrusion detection, including deep-based approaches or weightless neural networks, which feature surprising outcomes; ii) evaluate novel datasets (updated w.r.t. the obsolete KDD99 set) through a designed-fromscratch Python-based routine; iii) perform experimental analyses including time complexity and performance (accuracy and Fmeasure), considering both single-class and multi-class problems, and identifying trade-offs between resource consumption and performance. Our evaluation quantifies the value of neural networks, particularly when state-of-the-art datasets are used to train the models. This leads to interesting guidelines for security managers and computer network practitioners who are looking at the incorporation of neural-based ML into IDS.
\end{abstract}

Index Terms-Network Intrusion Detection, Neural Networks, Deep Learning, Network and Security Management

\section{INTRODUCTION}

Over the past few years, the adoption of Machine Learning (ML) techniques to the field of network security has become prominent [1], [2]. This is mainly due to the possibility of tackling a range of ever more sophisticated attacks able to circumvent security-based systems which rely on classic features inspection (e.g. port-control, signature-based flow detection, IPs black-listing, etc.). It is useful to recall that, especially in encrypted traffic analysis, the difference between deterministic and stochastic features is crucial. Deterministic ones pertains to "static" information embodied in security protocols such as TLS (e.g. record length, handshake types, cipher suites, etc.) [3], or IPSec (ISAKMP SPI Initiator/Responder, payload length, etc. ). On the other hand, stochastic features exploit the probabilistic nature (hard to hide in encrypted flows as well) of some traffic characteristics (e.g. the distribution of inter-arrival times). On behalf of ML-based techniques, it becomes quite

M. Di Mauro and G. Galatro are with the Department of Information and Electrical Engineering and Applied Mathematics (DIEM), University of Salerno, 84084, Fisciano, Italy (E-mail: mdimauro@unisa.it,giovanni.galatro@gmail.com).

A. Liotta is with the Free University of Bozen-Bolzano, Italy (E-mail: Antonio.Liotta@unibz.it) straightforward to manage stochastic features which, coupled with the deterministic ones, allow to characterize as accurately as possible an encrypted data flow.

Moreover, new network intrusion detection systems (NIDS) [4], [5] can actually interact with ML-based engines in order to learn the statistical features that characterize the various traffic flows and, in turn, classify them according to specific performance/time efficiency trade-offs. Among various possibilities of interaction, the Simple Network Management Protocol (SNMP) offers a flexible and standardized way to collect traffic data from a number of agents, by relying on Management Information Base (MIB) objects which provide information about some features to be managed $[6]-[8]$.

This notwithstanding, one of the biggest problems is to deal with the jungle of ML techniques which, depending on the underlying strategy, can exhibit completely different performance when applied in the intrusion detection field.

In fact, most attempts at surveying ML-based traffic classification problems have resulted in non-homogeneous comparisons and often unfair outcomes. Another flaw found in existing literature concerns the choice of a valid dataset. Most of studies, in fact, rely on the obsolete KDD99 dataset [9], or its evolved version NSL-KDD [10]. These datasets either do not contemplate essential features of modern data traffic (e.g. voice, video), or contain outdated signatures of network attacks [11].

In this paper we address the aforementioned shortcomings, providing the following main contributions. i) We survey neural-based techniques, which have recently taken prominence thanks to deep-based methods, in the specific context of traffic classification problems; we provide a critical comparison of algorithms that share a common paradigm (e.g. deep neural networks, linear vector quantization, etc.), including also techniques that are not typically applied to intrusion detection, such as weightless neural networks, whose results are quite unexpected. ii) We go beyond a traditional survey, providing an experimental-based assessment; we take into account novel traffic datasets (such as CIC-IDS-2017/2018), where both single-class cases (namely benign vs. malign) and multiclass cases (namely benign vs. $\operatorname{malign}_{1} \ldots$ vs. malign $_{k}$ ) are considered; we perform both performance analysis (through accuracy/F-measure figures) and time-complexity analysis.

The paper is organized as follows. Section II presents an excursus of related literature and, by means of a comparative table, we highlight differences and commonalities with other surveys. In Sect. III we review the most credited neuralbased methods. Section IV presents details about the novel considered datasets, where traffic features are grouped according to a similarity criterion. In Sect. $\mathrm{V}$ we provide an 
experimental-based comparison, where different neural-based techniques are juxtaposed through performance and timecomplexity analyses. Finally, Section VI concludes this work by also indicating some promising research directions.

\section{RELATED WORK ON ML TECHNIQUES APPLIED TO NETWORK INTRUSION DETECTION}

ML-based intrusion detection is becoming attractive for a variety of network environments including service providers [12], sensor networks [13], Internet of Things [14], and automotive [15]. This notwithstanding, a great part of scientific literature, which faces the problem of data traffic classification through machine learning techniques, suffers from the datasets obsolescence issue. For many years, the only dataset available to the scientific community was the so-called KDD99, which is still broadly used today to validate ML-based algorithms and techniques when dealing with traffic classification problems. Unfortunately, this is an outdated 20-years-old dataset involving network attacks that have already been mitigated. An example is satan probing, an attack aimed at probing a computer for security loophole, based on the satan tool that was created at the end of the $1990 \mathrm{~s}$, but is today no longer documented as a Web page.

Other examples include: warezmaster/warezclient, which exploits some old vulnerabilities of the anonymous FTP protocol, and smurf attacks, aimed at attaining default router settings that allowed directed broadcasts. Yet, currently, router vendors simply deactivate this functionality. An ameliorated version of KDD99 is known as NSL-KDD. However, despite providing some improvements (e.g. no redundant records, better balancing between training and test set), NSL-KDD is still not taking into account crucial information that characterizes novel cyber attacks.

This notwithstanding, both KDD99 and NSL-KDD datasets are still broadly used to test some functionalities of NIDS systems that implement neural-based techniques. For instance, in $[16]-[18]$ the authors show the effectiveness of using an NIDS in conjunction with an artificial neural network (ANN) to improve the quality of traffic detection, where a validation stage onto the KDD99 dataset is performed. A deep learning approach is used in [19], based on KDD99, to verify accuracy against an SVM methodology. A novel approach based on ANN (referred to as self-taught learning) is applied in [20] to enable an NIDS to detect previously unseen attacks via reconstructions made on unlabeled data. This work provides tests on both KDD99 and NSL-KDD. In [21] and [22] the authors adopt neural-based methods exploiting Self-Organizing Maps. In [23] and [24], Learning Vector Quantization is coupled with SVM and k-Nearest Neighbor, respectively, to detect traffic anomalies. In [25]-[28] deep neural networks concepts are applied to intrusion detection systems.

The KDD99 and NSL-KDD datasets have also been used to test a variety of non neural-based techniques such as Support Vector Machine [29]-[33], Principal Component Analysis [34]-[38], Decision Trees [39]-[42], [42], [43], and various unsupervised approaches [44]-[49].

By contrast to the aforementioned works, some recent datasets are emerging from new testbeds which adhere more strictly to real-world network scenarios. For instance, the Cyber Range Lab of the Australian Centre for Cyber Security provides two recent datasets: the UNSW-NB15 dataset [50] which includes a mix of legitimate network activities and synthetic attacks, and the Bot-IoT dataset [51] which embeds normal and simulated network traffic gathered in an IoTcompliant testbed, along with various types of attacks.

Again, the datasets recently released by the Canadian Institute for Cybersecurity (CIC) [52] represent the state-of-the-art, in terms of both complexity and novelty of network attacks. These datasets have been created starting from an experimental testbed under controlled conditions [11], whereby an attack network (including a router, a switch, and four PCs equipped with Kali Linux OS, which is a popular Linux distribution to perform penetration testing) is counterposed to a victim network (including routers, firewalls, switches, and various PCs equipped with Windows, Linux, and MacOS operating systems). An evolved version of this testbed has been designed to run on Amazon AWS [53]. In this case, the attacking infrastructure includes $50 \mathrm{PCs}$, and the victim network includes 420 PCs and 30 servers. The implemented attacks encompass Distributed Denial of Service (DDoS), Portscan, Bruteforce, along with some typical Android-based network attacks injecting malicious codes such as adwares, malwares, and ransomwares.

The interest for such novel datasets is proven by novel works as detailed in the following. In [54], authors validate an artificial neural network system onto a CIC-released dataset to detect the malicious traffic generated by a botnet, out of the regular traffic. A hybrid neural network to detect anomalies in network traffic is proposed in [55], where the CIC-IDS2017 dataset has been exploited. Specifically focused on DDoS detection is the work in [56], where a neural-based approach relying on the implementation of a simple Multi-Layer Perceptron is contrasted to the Random Forest technique. Again focused on DDoS detection is [57], where some classic MLbased techniques (e.g. Naïve Bayes and Logistic Regression) are used to distinguish regular traffic from malicious one.

Going more precisely into the set of papers that share with the proposed work the purpose of surveying and/or comparing ML techniques for intrusion detection, we have gathered the prominent papers in Table II The first column contains a pointer to the source; the second column reports the type of experimental analysis (if any); the third column highlights the type of datasets utilized (single/multi-class); the last column provides a brief description of the surveyed material, whereby the qualification "non-homogeneous" refers to a comparison among techniques belonging to different families, thus, hardly comparable.

Going beyond the adoption of novel datasets, we want to highlight the two most significant differences arising between the proposed review and the technical literature: First, to avoid dispersions, we prefer to focus on a specific family of ML techniques, namely the neural networks, so to guarantee more fair comparisons among outcomes. Such a focus allows us to better reveal the surprising behavior of weightless neural networks (traditionally exploited in the field of image classification) which exhibits an extraordinary advan- 
TABLE I: Prominent Related Work surveying ML techniques applied to Network Intrusion Detection.

\begin{tabular}{|c|c|c|c|}
\hline Authors & Experiments & Single/Multi Class & Description \\
\hline Nguyen et al. $|58|$ & N/A & N/A & $\begin{array}{l}\text { Classic survey on ML-based techniques with } \\
\text { pointers to other works but with no experi- } \\
\text { ments. }\end{array}$ \\
\hline Boutaba et al. |59| & N/A & N/A & $\begin{array}{l}\text { Survey on ML-based techniques applied to var- } \\
\text { ious networking-related problems (from traffic } \\
\text { classification to routing or QoS/QoE manage- } \\
\text { ment). }\end{array}$ \\
\hline Hindy et al. $|60|$ & N/A & N/A & $\begin{array}{l}\text { Survey on IDS techniques taking into account } \\
\text { ML algorithms such as ANN, K-means and } \\
\text { SVM. }\end{array}$ \\
\hline Khraisat et al. [61] & N/A & N/A & $\begin{array}{l}\text { Survey on Signature and Anomaly-based IDS } \\
\text { techniques applying ML methods on NSL- } \\
\text { KDD dataset. }\end{array}$ \\
\hline Aldweesh et al. |62| & N/A & N/A & $\begin{array}{l}\text { Survey on Deep Learning techniques for IDSs } \\
\text { with pointers to other works but with no ex- } \\
\text { periments. }\end{array}$ \\
\hline Fernandes et al. |63| & N/A & N/A & $\begin{array}{l}\text { Survey on various techniques (ML, Statistical, } \\
\text { Information Theory) for intrusion detection } \\
\text { with pointers to other works but with no ex- } \\
\text { periments. }\end{array}$ \\
\hline Buczak et al. [64] & N/A & N/A & $\begin{array}{l}\text { Survey on Data Mining and ML methods for } \\
\text { intrusion detection with pointers to other works } \\
\text { but with no experiments. }\end{array}$ \\
\hline Tidjon et al. $|65|$ & N/A & N/A & $\begin{array}{l}\text { Survey on various techniques (mainly ML- } \\
\text { based) to be applied in intrusion detections } \\
\text { with pointers to other works but with no ex- } \\
\text { periments. }\end{array}$ \\
\hline Azwar et al. |66 & Performance analysis & Single Class & $\begin{array}{l}\text { Non-homogeneous comparisons among vari- } \\
\text { ous approaches (trees, NN) by using modern } \\
\text { CIC-IDS17 dataset. }\end{array}$ \\
\hline Moustafa et al. [67] & Performance analysis & Single Class & $\begin{array}{l}\text { Holistic Survey on ML methods with experi- } \\
\text { ments on feature reduction techniques (ARM, } \\
\text { PCA, ICA). }\end{array}$ \\
\hline Meena et al. |68| & Time analysis & Single Class & $\begin{array}{l}\text { Non-homogeneous comparisons between J48 } \\
\text { and Naïve Bayes techniques on KDD and } \\
\text { NSL-KDD datasets. }\end{array}$ \\
\hline Rama et al. |69| & $\begin{array}{l}\text { Performance analysis, } \\
\text { Time analysis (partial) }\end{array}$ & Single Class & $\begin{array}{l}\text { Non-homogeneous comparisons among vari- } \\
\text { ous algorithms (e.g. J48, Naïve Bayes, Bag- } \\
\text { ging) on KDD and NSL-KDD datasets. }\end{array}$ \\
\hline Yin et al. |70| & Performance analysis & Single/Multi Class & $\begin{array}{l}\text { Non-homogeneous comparisons among vari- } \\
\text { ous and different approaches (e.g. J48, ANN, } \\
\text { SVM) on KDD99 dataset. }\end{array}$ \\
\hline This work & $\begin{array}{l}\text { Performance analysis, } \\
\text { Time analysis }\end{array}$ & Single/Multi Class & $\begin{array}{l}\text { Homogeneous comparisons among neural- } \\
\text { based approaches (Deep, Weightless NN, } \\
\text { LVQ, SOM) performed on modern CIC-IDS- } \\
\text { 2017/2018 datasets. }\end{array}$ \\
\hline
\end{tabular}

tageous accuracy/time complexity trade-off w.r.t. other NNbased methods. Then, we carry out an experimental analysis including performance and time complexity (this latter often neglected in technical literature) about all the described NN techniques; this effort overcomes the limit of gathering findings from various works (where different authors exploit different testbeds exhibiting different performance), thus, it results in a uniform vision of neural methods in the field of intrusion detection. Accordingly, Table 1 helps pinpointing such novel aspects of the present paper, as per last row.

\section{NEURAL-BASED TECHNIQUES UNDER SCRUTINY}

In this section we offer a brief recap of neural-based techniques that we then employ in Section V to perform our comparative assessment. We start with Multi-Layer Perceptron (MLP) which represents the basis for implementing ANNs and deep neural networks (DNN). Then, we consider WiSARD, one of the most representative algorithms of weightless neural networks. These typically provide interesting performance results, but have traditionally been applied in domains such as image classification rather than intrusion detection. We 
examine Learning Vector Quantization (LVQ) methods (with 3 variants), where the notion of codebook vector will be introduced. Finally, we take into account the Self-Organizing Maps (SOM) technique.

\section{A. Multi-Layer Perceptron}

The Multilayer Perceptron is one of the most representative type of feedforward-based ANNs, whereby there are no cycles arising in the connections among nodes. MLPs exhibit a fully connected structure of neurons, where each individual neuron calculates the weighted sum of $n$ inputs, adds a bias term $b$, and then applies an activation function $\phi(\cdot)$ to produce the output $s$, namely

$$
s=\phi\left(\sum_{i=1}^{n} w_{i} x_{i}+b\right) .
$$

Figure 1 depicts $i$ ) (left panel) the model of a single neuron (or single-unit perceptron) implementing (1), and ii) (middle panel) a simplified structure of a typical MLP model with 5 neurons in the input layer, 3 neurons in the hidden layer, and 1 neuron in the output layer. In case of multiple hidden layers, MLP implements a Deep Neural Network (DNN) [71] as reported in the right panel.

The basic MLP functioning is described next. Input $I_{n}$ (see Fig. 1 - middle panel) activates the hidden layer (or layers) by following the forward activation direction, which is what justifies the term feed-forwarding neural network. Similarly, neurons in the hidden layers feed forward into output neurons, thus, an output value is obtained. It is useful to recall that the activation function is aimed at deciding whether or not a neuron would be activated, introducing some non-linearity into the neuron output. A variety of activation functions exist [72] including: step function, linear, sigmoid, hyperbolic tangent, ReLU (Rectified Linear Unit), Softmax and many others.

The MLP training stage is achieved through backpropagation, a mechanism exploited to adjust weights of neurons aimed at progressively minimizing the error through Gradient Descent (GD), an iterative optimization algorithm useful to find local minima. Precisely, the purpose is to minimize an error function (e.g. least squares):

$$
\mathcal{E}=\sum_{p \in \mathcal{P}}\left\|t_{p}-s_{p}\right\|^{2},
$$

where $\mathcal{P}$ is the set of training patterns, $t_{p}$ is the target, and $s_{p}$ is the output for the example pattern $p$. The weight-updating rule, used to progressively compute the new weight $w_{\text {new }}$, is derived by evaluating the gradient $\partial \mathcal{E} / \partial w$, so that:

$$
\begin{array}{r}
w_{\text {new }}=w+\Delta w \\
\Delta w=-\eta \frac{\partial \mathcal{E}}{\partial w}+\alpha \Delta w_{\text {prev }}
\end{array}
$$

where: i) $\eta$ is the learning rate, namely a hyperparameter lying in the range $(0,1)$ associated to the step size of the GD procedure (N.B.: too small $\eta$ implies difficulty of convergence, whereas too large $\eta$ could result in indefinite oscillations); ii) $\alpha$ is defined as the momentum, a term lying in the range
$(0,1)$ used to weight the influence of each iteration. It is worth noting that, since derivative operations are involved in the backpropagation algorithm, non-linear activation functions (e.g. Sigmoid, ReLU) have to be exploited, whose derivative functions exist and are finite.

In our MLP-based experiment we use two types of activation functions: ReLU for all the layers except for the output, and Softmax for the neurons in the output layer. ReLU has been proven to be one of the most effective activation functions when dealing with deep neural networks [73], [74] since it allows the whole network to converge rapidly. Conversely, Softmax is particularly suited for handling multiple classes in the output stage [75].

\section{B. Evolved Deep architectures}

Deep learning approaches allow to face a problem in a hierarchical way. Lower layers of the model are associated to a basic representation of the problem, whereas higher layers encode more complex aspects. Inputs feeding each layer of a DNN are manipulated through transformations which are parametrized by a number of weights. Although deep approaches are very promising, two main issues remain opened: first, training these architectures requires a significant computational power, and, then, the huge number of hyperparameters makes the tuning process very hard. In the following, we briefly discuss the most recent architectures relying on a deep-based approach.

Convolutional Neural Networks (CNNs): Such a technique [76] takes inspiration from the human visual cortex, which embodies areas of cells responsive to particular regions of the visual field. This structure makes CNNs exceptionally suited for applications such as images classification or objects detection. Two main stages characterize the CNN lifecycle: feature extraction and classification. In the first stage, the socalled convolutional filters extract multiple features from the input and encode them in feature maps. The "convolution" is the mathematical operation consisting in an element-wise product and sum between two matrices, and has the main drawback of being hugely time consuming. The output of each convolutional layer feeds the activation function layer (e.g. ReLU) which produces an activation map by starting from a feature map. Finally, an optional pooling layer keeps only significant data. On the other hand, the classification stage is composed of a number of fully connected (or dense) layers followed by a Softmax output layer.

Recurrent Neural Networks (RNNs): It is a class of DNNs conceived on the basis of a work of Rumelhart [77], explicitly designed to deal with sequential data. Thus, RNNs are well suited for modelling language (intended as a sequence of interconnected words) in the field of the so-called natural language processing (NLP). The key concept in RNNs is the presence of cycles, which represent the internal memory exploited to evaluate current data with respect to the past. Such a temporal dependency calls for the introduction of time-based hidden states obeying to:

$$
h(t)=\phi(h(t-1), x(t)),
$$



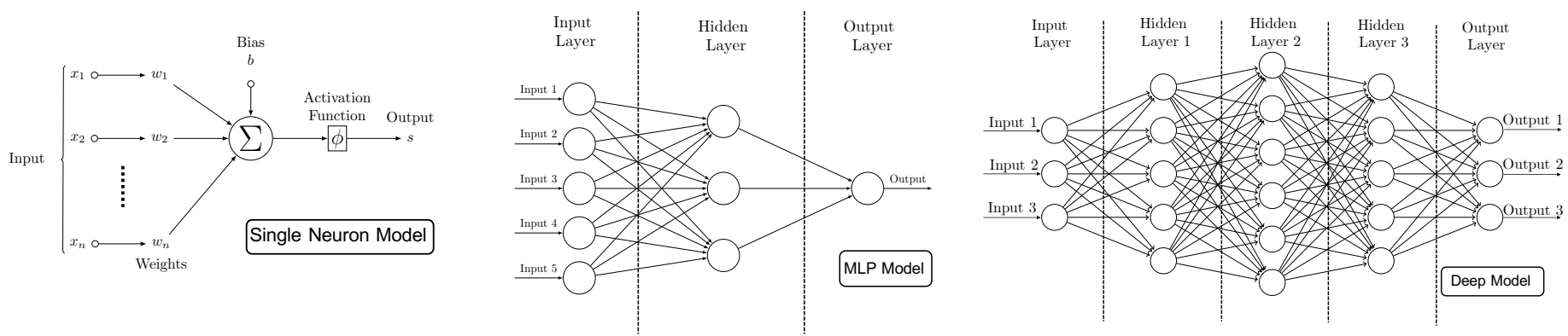

Fig. 1: Single Neuron model (left panel). MLP model (middle panel). Deep NN model (right panel)

with the meaning that an internal state $h$ at time $t$ can be represented in terms of the input at time $t$ and the previous hidden state at time $t-1$. In this way, an RNN is helpful to predict the next element of a time series, or the next word in a sentence based on the number of the previous words. One of the main drawbacks of RNNs is dealing with long-term dependencies connected with transferring information from earlier to later times steps across too long sequences. To tackle this issue, two more sophisticated variants of RNNs have been introduced: LSTM and GRU.

Long Short-Term Memory (LSTM): It is a special RNN architecture [78] conceived to learn long-term dependencies, namely, to store information for a long period of time. Basically, an LSTM unit (replacing an ordinary node in the hidden layer) is represented by a cell state in charge of carrying the information, and by three different gates aimed at regulating the information flow: forget, input, and output gates. The forget gate decides what information keep or discard on the basis of a forgetting coefficient calculated by input data $x(t)$ and the previous hidden state $h(t-1)$; the input gate decides how to update the cell state; the output gate decides which information has to be passed to the next unit, on the basis of input data and the previous hidden state. For the $i$-th LSTM unit, the hidden state at time $t$ can be expressed as:

$$
h^{i}(t)=o u t^{i}(t) \cdot \tanh \left(c^{i}(t)\right),
$$

where out $^{i}(t)$ is an output gate which tunes the amount of memory, and $c^{i}(t)$ represents the cell state of LSTM unit $i$ at time $t$.

Gated Recurrent Unit (GRU): It is a lightweight version of LSTM [79] and has only two gates: update gate and reset gate. The former plays a similar role of forget and input gates of LSTM; the latter is exploited to decide how many past data to forget. In the GRU model, the hidden state $h^{i}(t)$, corresponding to the $i$-th GRU unit, can be expressed as a linear interpolation between the hidden state at time $t-1$ and the candidate activation (or hidden state) $\widetilde{h^{i}}(t)$ viz.

$$
h^{i}(t)=\left(1-z^{i}(t)\right) h^{i}(t-1)+z^{i}(t) \widetilde{h^{i}}(t)
$$

where $z^{i}(t)$ is the update gate which decides about the updating amount of its candidate activation.

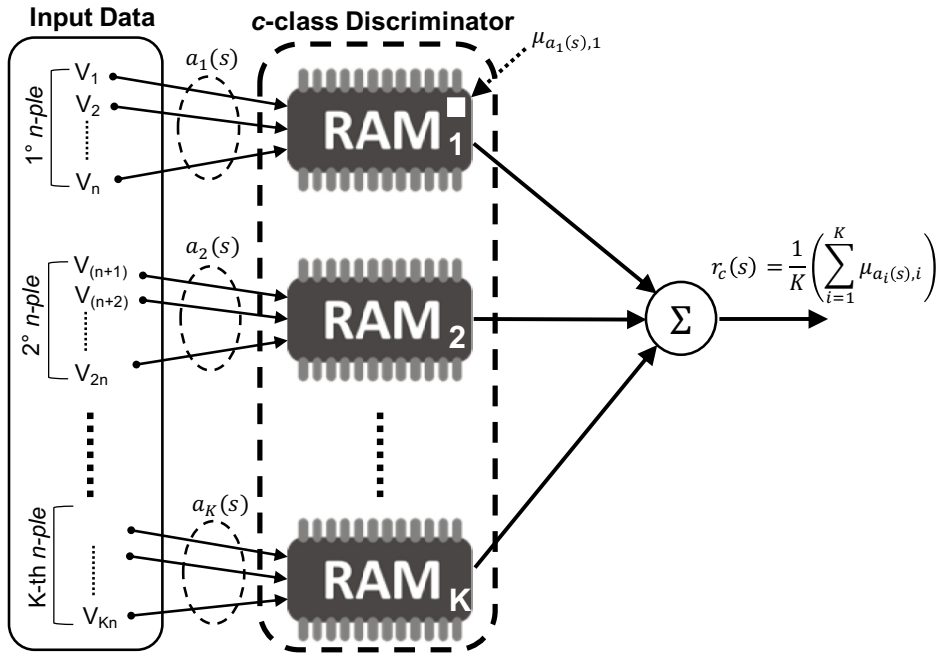

Fig. 2: Model of a single class discriminator for the WiSARD algorithm.

\section{WiSARD}

WiSARD ${ }^{1}$ is a supervised method [80], [81] that was originally conceived for image classification, but has recently been proven to be effective in more general multi-class problems.

WiSARD falls under the class of weightless neural networks (WNNs) since it exploits lookup tables, instead of weights, to store the functions evaluated by the individual neurons. WNNs rely on a mechanism inspired to the random access memory (RAM) encoding functionalities, since input data are transformed into binary. This process has a noteworthy benefit in terms of time complexity due to the use of Boolean logic, which can be further improved by exploiting pipelining and parallelism. In short, WiSARD is composed of a set of classifiers (or discriminators), each one in charge of learning binary patterns associated to a particular class. In turn, a discriminator is composed of a set of neurons, referred to as RAM neurons as depicted in Fig. 2. Similarly to conventional RAM circuits, a RAM neuron (a.k.a. $n$-tuple neuron) can be interpreted as a RAM having $2^{n}$ memory locations addressed by $n$ address lines (inputs) representative of neuron connectivity.

During the training stage, each RAM neuron learns the occurrences of $n$-tuple vectors extracted from a training pattern, and stores them in a memory cell (equivalent to a RAM writing

\footnotetext{
${ }^{1}$ Wilkes, Stonham and Aleksander Recognition Device
} 
operation). Precisely, given $\mu_{a, i}$ the memory cell with address $a$ in the $i$-th RAM (initially empty), the following update rule holds:

$$
\mu_{a, i}=\theta\left(\sum_{p \in \mathcal{P}} \delta_{a, a_{i}(p)}\right),
$$

where: function $\theta(z)$ amounts to $z$ if $0 \leq z \leq 1$ and to 1 if $z>1 ; p$ is a pattern defined in the training set $\mathcal{P} ; a_{i}(p)$ is the address generated starting from pattern $p ; \delta$ is the Kronecker delta function, amounting to 1 if $a=a_{i}(p)$ and 0 elsewhere.

The classification stage (equivalent to a RAM reading operation) consists of classifying an unseen pattern $s$ by assigning $s$ to a class $c$, provided that the correspondent discriminator exhibits the highest output, namely

$$
\underset{c}{\arg \max }\left(\sum_{i=1}^{K} \mu_{a_{i}(s), i}\right),
$$

whereas, the response of the $c$-th class discriminator on pattern $s$ is:

$$
r_{c}(s)=\frac{1}{K} \sum_{i=1}^{K} \mu_{a_{i}(s), i} .
$$

Accordingly, since WiSARD is made of a set of discriminators, the overall response of a set of discriminators trained on $N$ classes produces a response vector $\mathbf{r}(s)=\left[r_{c_{1}}(s), \ldots, r_{c_{N}}(s)\right]$.

\section{Learning Vector Quantization}

Learning Vector Quantization (LVQ) [82] directly stems from classical Vector Quantization, a signal-approximation method aimed at representing the input data vectors $x \in \mathbb{R}^{n}$ through a finite number of codebook vectors $m_{i} \in \mathbb{R}^{n}, i=$ $1,2, \ldots, k$. The goal is to find the codebook vector $m_{v}$ that best approximates $x$, usually in terms of Euclidean distance, namely:

$$
v=\underset{i}{\arg \min }\left\|x-m_{i}\right\| .
$$

The main purpose of LVQ is to define class regions (over the input data space), each one containing a subset of a similarly labeled codebook. Accordingly, it is possible to pinpoint hyperplanes between neighboring codebook vectors defining the so-called quantization regions. By assuming that all samples of $x$ derive from a finite set of classes $S_{k}$, the idea is to $i$ ) assign a subset of codebook vectors to each class $S_{k}$, and ii) search for $m_{v}$ having the smallest Euclidean distance from $x$. Different versions of LVQs have been introduced in the literature with slight differences, as introduced in the following.

1) LVQ1: Assume that $x(t)$ is an input sample and $m_{i}(t)$ contains sequential values of $m_{i}(t=0,1,2, \ldots)$. The LVQ1 algorithm allows to find values of $m_{i}$ in 10 that asymptotically minimize errors, and is defined by the following set of equations:

$$
\begin{aligned}
m_{v}(t+1) & =m_{v}(t)+\alpha(t)\left[x(t)-m_{v}(t)\right], \\
m_{i}(t+1) & =m_{i}(t),
\end{aligned}
$$

where (11) refers both for cases that $x$ and $m_{v}$ belong to the same or different classes, 12 holds for $i \neq v$, and $\alpha(t)$ is the learning rate. This algorithm admits also an optimized version (a.k.a. OLVQ1), where an individual learning rate $\alpha_{i}$ is assigned to $m_{i}$, thus, basic $m_{v}(t+1)$ equation in (12) becomes:

$$
m_{v}(t+1)=\left[1-c(t) \alpha_{v}(t)\right] m_{v}(t)+c(t) \alpha_{v}(t) x(t),
$$

where $c(t)=+1[-1]$ if the classification is correct [wrong].

2) $L V Q 2$ : Differently from the standard procedure implemented in LVQ1, here two codebook vectors $m_{i}$ and $m_{j}$ (belonging to the correct and to the wrong class, respectively) are simultaneously updated. In this case, $x$ must fall within a "window" defined around the hyperplanes of $m_{i}$ and $m_{j}$. The correspondent algorithm is defined by the following set of equations:

$$
\begin{aligned}
& m_{i}(t+1)=m_{i}(t)-\alpha(t)\left[x(t)-m_{i}(t)\right], \\
& m_{j}(t+1)=m_{j}(t)-\alpha(t)\left[x(t)-m_{j}(t)\right],
\end{aligned}
$$

where (14) holds when $x$ and $m_{i}$ belong to the same class, (15) holds when $x$ and $m_{i}$ belong to different classes, and with $m_{i}$ and $m_{j}$ being the two closest codebook vectors to $x$. Moreover, $x$ has to fall within a window of relative width $w$ if

$$
\min \left(\frac{e_{i}}{e_{j}}, \frac{e_{j}}{e_{i}}\right)>k,
$$

where $e_{i}$ and $e_{j}$ represent, respectively, the Euclidean distances of $x$ from $m_{i}$ and $m_{j}$, and $k=\frac{1-w}{1+w}$.

3) LVQ3: This variant of LVQ2 admits the same set of equations (14), (15), with the difference that the learning rate is weighted with a parameter $\epsilon$, whose best values are found empirically to lie in the [0.1-0.5] interval of values:

$$
m_{h}(t+1)=m_{h}(t)+\epsilon \alpha(t)\left[x(t)-m_{h}(t)\right],
$$

for $k \in i, j$ if $x, m_{i}$, and $m_{j}$ belong to the same class.

\section{E. Self-Organizing Maps}

SOM takes inspiration from a particular adaptive characteristic that allows the human brain to empower the experience. Specifically, given a physical stimulus (namely, the input) which activates multiple neurons of a certain brain area in parallel, those neurons that are more sensitive to the input stimulus will go about influencing all other neighboring neurons.

This biological mechanism has led to designing SOM as a nonlinear mapping of high-dimensional input data onto elements of a low-dimensional array (a.k.a. lattice) [83], according to a principle known as competitive learning. This is different from the classic ANN-based approach, where weights are updated to iteratively minimize errors. In competitive learning, several neurons are fed with the same input (in parallel) and compete to become the possible "winner" in relation to that particular input.

According to this strategy, and assuming that the weight vector of neurons has the same dimensionality as the input, the output neuron activation increases with larger similarity between the weight vector of the neuron and the input [84]. 
Precisely, by considering a network composed of $k$ neurons (with $k<<n$ being $n$ the data set size), an input vector $x=\left[x_{1}, x_{2}, \ldots, x_{n}\right]^{T} \in \mathbb{R}^{n}$ and a reference (or weight) vector $m_{i}=\left[m_{i 1}, m_{i 2}, \ldots, m_{i n}\right]^{T} \in \mathbb{R}^{n}$ associated with neuron $i$, the competitive approach can be summarized according to the following steps:

i) Compute the smallest Euclidean distance $d=\arg \min _{i}$ $\left\|x-\mathrm{m}_{i}\right\| \forall i \in(1, \ldots, m)$ having the meaning of the activation value for the $i$-th neuron. The neuron having $d$ is declared as winner.

ii) The $i$-th neuron is updated according to the following rule: $m_{i}(t+1)=m_{i}(t) h(t)\left[x(t)-m_{i}(t)\right]$ where $t=$ $(0,1, \ldots)$ is an integer discrete time reference, whereas $h(t)$ is the neighborhood function defined over the lattice points that is typically implemented through a Gaussian kernel (the same as then one exploited for the present experimental analysis):

$$
h(t)=\alpha(t) \cdot \exp \left(-\frac{\left\|\ell_{c}-\ell_{i}\right\|^{2}}{2 \sigma^{2}(t)}\right),
$$

where: $\alpha(t)$ is the learning rate factor, $\ell_{c} \in \mathbb{R}^{2}$ and $\ell_{i} \in$ $\mathbb{R}^{2}$ are, respectively, the location vectors of neurons $c$ and $i$ mapped on the lattice structure (supposed to be bi-dimensional), and $\sigma(t)$ represents the kernel width.

Although SOM may be considered as the unsupervised counterpart version of LVQ, a common way to exploit SOM for classification purposes is to consider a supervised version (sometimes dubbed as LVQ-SOM [83] and implemented in our analysis) relying on the following consideration: once we know that each training sample $x(t)$ and $m_{i}(t)$ have been assigned to specific classes, $h(t)$ has to be set to positive if $x(t)$ and $m_{i}(t)$ belong to the same class, and negative if they belong to different classes, by taking into account that such a rule is applied for each $m_{i}(t)$ in the neighborhood of the winner.

\section{THE EXPERIMENTAL DATASETS}

In this section we present further details about datasets employed in our experimental analysis. As already remarked in Section II] the datasets must convey the most recent information about a variety of cyber attacks across data networks. We adopted datasets released from CIC [52], and precisely: the $D D o S$ dataset, containing traffic relating to distributed denial of service attacks designed to saturate network resources; the Portscan dataset, including attempts of Portscan, a technique used to discover open ports on network devices; the WebAttack dataset which encompasses various malicious traffic ranging from Cross-Site Scripting to Sql Injection; the TOR dataset, a collection of network traffic traversing the anonymous TOR circuit often conveying malicious information; and the Android dataset, embedding a number of mobile (Android-based) adwares.

These datasets have been used to perform two kinds of neural-based analyses: a single-class analysis, aimed at classifying the network traffic as benign or malign (binary information); and a multi-class analysis, aimed at distinguishing more classes of attacks. Each dataset has been cleaned and
TABLE II: Synthetic description of adopted features

\begin{tabular}{|c|c|}
\hline Family & List of features \\
\hline Coarse-Grained & $\begin{array}{l}\text { 1. Source IP Address } \\
\text { 2. Destination IP Address } \\
\text { 3. Source Port } \\
\text { 4. Destination Port } \\
\text { 5. Transport Protocol Type }\end{array}$ \\
\hline Time-Based & $\begin{array}{l}\text { 6. Flow duration } \\
\text { 7. Average inter-arrival times (IAT) between two flows } \\
\text { 8. IAT standard deviation (std) between two flows } \\
\text { 9. IAT max between two flows } \\
\text { 10. IAT min between two flows } \\
\text { 11. IAT tot between two pkts sent in fwd direction } \\
\text { 12. IAT avg between two pkts sent in fwd direction } \\
\text { 13. IAT std between two pkts sent in fwd direction } \\
\text { 14. IAT max between two pkts sent in fwd direction } \\
\text { 15. IAT min between two pkts sent in fwd direction } \\
\text { 16. IAT tot between two pkts sent in bwd direction } \\
\text { 17. IAT avg between two pkts sent in bwd direction } \\
\text { 18. IAT std between two pkts sent in bwd direction } \\
\text { 19. IAT max between two pkts sent in bwd direction } \\
\text { 20. IAT min between two pkts sent in bwd direction } \\
\text { 21. Avg time a flow was active before becoming idle } \\
\text { 22. Std time a flow was active before becoming idle } \\
\text { 23. Min time a flow was active before becoming idle } \\
\text { 24. Max time a flow was active before becoming idle } \\
\text { 25. Avg time a flow was idle before becoming active } \\
\text { 26. Std time a flow was idle before becoming active } \\
\text { 27. Min time a flow was idle before becoming active } \\
\text { 28. Max time a flow was idle before becoming active }\end{array}$ \\
\hline Flow-Based & $\begin{array}{l}\text { 29. Flow byte rate } \\
\text { 30. Flow pkt rate } \\
\text { 31. Avg no. of pkts in a sub-flow in fwd direction } \\
\text { 32. Avg no. of pkts in a sub-flow in bwd direction } \\
\text { 33. Avg no. of bytes in a sub-flow in fwd direction } \\
\text { 34. Avg no. of bytes in a sub-flow in bwd direction }\end{array}$ \\
\hline Packet-Based & $\begin{array}{l}\text { 35. Tot pkts in the fwd direction } \\
\text { 36. Tot length of pkts in the fwd direction } \\
\text { 37. Avg length of pkts } \\
\text { 38. Std length of pkts } \\
\text { 39. Variance length of pkts } \\
\text { 40. Avg length of pkts in the fwd direction } \\
\text { 41. Std length of pkts in the fwd direction } \\
\text { 42. Max length of pkts in the fwd direction } \\
\text { 43. Min length of pkts in the fwd direction } \\
\text { 44. Tot pkts in the bwd direction } \\
\text { 45. Tot length of pkts in the bwd direction } \\
\text { 46. Avg length of pkts in the bwd direction } \\
\text { 47. Std length of pkts in the bwd direction } \\
\text { 48. Max length of pkts in the bwd direction } \\
\text { 49. Min length of pkts in the bwd direction } \\
\text { 50. Avg no. of pkt bulk rate in fwd direction } \\
\text { 51. Avg no. of pkt bulk rate in bwd direction } \\
\text { 52. Fwd pkt rate } \\
\text { 53. Bwd pkt rate } \\
\text { 54. Min segment size in fwd direction }\end{array}$ \\
\hline Byte-Based & $\begin{array}{l}\text { 55. Avg no. of byte rate in fwd direction } \\
\text { 56. Avg no. of byte rate in bwd direction } \\
\text { 57. No. of bytes sent in init win in fwd direction } \\
\text { 58. No. of bytes sent in init win in bwd direction } \\
\text { 59. Tot bytes used for headers in fwd direction } \\
\text { 60. Tot bytes used for headers in bwd direction }\end{array}$ \\
\hline Flag-Based & $\begin{array}{l}\text { 61. No. of times URG flag set in fwd direction } \\
\text { 62. No. of times PSH flag set in fwd direction } \\
\text { 63. No. of times FIN flag set in fwd direction } \\
\text { 64. No. of times SYN flag set in fwd direction } \\
\text { 65. No. of times RST flag set in fwd direction } \\
\text { 66. No. of times ACK flag set in fwd direction } \\
\text { 67. No. of times URG flag set in bwd direction } \\
\text { 68. No. of times PSH flag set in bwd direction } \\
\text { 69. No. of times FIN flag set in bwd direction } \\
\text { 70. No. of times SYN flag set in bwd direction } \\
\text { 71. No. of times RST flag set in bwd direction } \\
\text { 72. No. of times ACK flag set in bwd direction } \\
\text { 73. PSH flag count } \\
\text { 74. FIN flag count } \\
\text { 75. SYN flag count } \\
\text { 76. RST flag count } \\
\text { 77. ACK flag count } \\
\text { 78. ECE flag count }\end{array}$ \\
\hline
\end{tabular}


TABLE III: Optimized hyperparameters for the exploited algorithms.

\begin{tabular}{|c|c|}
\hline Algorithm & Optimized hyperparameters and models info \\
\hline $\begin{array}{l}\text { MLP-1, Deep-2, } \\
\text { Deep-3 }\end{array}$ & $\begin{array}{l}\text { Stochastic Gradient Descent with adaptive hyperparams. } \\
\text { (Adam version }-[85 \mid) \\
\text { - LR (learn. rate) }=0.001 \\
\text { - Number of weights }=2000 \\
\text { - Exp. Decay (first moment estimate) }=0.9 \\
\text { - ReLU activation function } \\
\text { - Neurons per hidden layer: MLP-1(26); Deep-2(23,10); Deep- } \\
3(20,16,11)\end{array}$ \\
\hline Convolutional & $\begin{array}{l}7 \text { filters }(4 x 1), 8 \text { neurons fully connected } \\
\cdot 1 \text { Pooling layer with Pooling size }=2 \\
\cdot 1 \text { Dropout layer with Dropout rate }=0.3 \\
\cdot \text { ReLU activation function }\end{array}$ \\
\hline Recurrent-type & $\begin{array}{l}\text { 18 Recurrent units (RNN), } 10 \text { neurons fully connected } \\
\text { - } 6 \text { LSTM units, } 8 \text { neurons fully connected } \\
.8 \text { GRU units, } 10 \text { neurons fully connected }\end{array}$ \\
\hline WiSARD & $\begin{array}{l}\cdot \text { Batch Size }=100 \\
\text { Resolution (in bit) per neuron: } 8\end{array}$ \\
\hline $\operatorname{LVQ}(1,2,3)$ & $\begin{array}{l}\cdot \text { Batch Size }=100 \\
\cdot \mathrm{LR}=0.3 \\
\cdot \text { Codebook Vectors }=20 \\
\cdot \text { Window Size (for LVQ2 and LVQ3) }=0.3 \\
\cdot \epsilon \text { (for LVQ3) }=0.1\end{array}$ \\
\hline SOM & $\begin{array}{l}\cdot \text { Batch Size }=100 \\
\cdot \text { LR=0.3 } \\
\cdot \text { Hexagonal Topology with Neighborhood Size }=8 \\
\cdot \text { Neighborhood Function: Gaussian }\end{array}$ \\
\hline
\end{tabular}

re-balanced through a Python routine designed from scratch, and contains $2 \cdot 10^{4}$ instances and 78 features, which are then grouped in 6 macro-classes, as indicated in the following (refer to the Table II for an exhaustive list of features):

- Coarse-grained features: Source and Destination Port, Protocol Type, Source and Destination IP Address;

- Time-based features: Backward/Forward inter-arrival times between two flows, duration of active flow (mean, std, min, max), duration of an idle flow (mean, std, min, $\max )$, etc.;

- Flow-based features: Length of a flow (mean, min, max, etc.);

- Packet-based features: Backward/Forward number of packets in a flow, Backward/Forward length of packets in a flow (mean, std, min, max, etc.);

- Byte-based features: Backward/Forward number of bytes in a flow, Backward/Forward number of bytes used for headers, etc.;

- Flag-based features: Number of packets with active TCP/IP flags (SYN, FIN, PUSH, RST, URG, etc.). Please note that, for example, feature 62 (68) indicates the no. of times the PSH flag is set in the forward (backward) direction, whereas feature 73 indicates the overall number of packets containing such a flag.
These features allow to derive statistical information that cannot be hidden in a possible malicious flow. Let us consider, for instance, a DDoS attack. This is typically designed to overwhelm the resources of a target network [86], [87] by conveying legitimate information (e.g. trivial HTTP requests in case of an application-layer DDoS attack) which would pass unnoticed to a classic signature-based detection systems. However, DDoS attacks are designed to be a coordinated effort where multiple, malicious entities (a.k.a. bots) send few, tiny packets to the target. Similarly, the structure of a Portscan attack, characterized by a very quick scan of the victim's destination port by the network attacker, can be learned by crossing information of destination port and timebased information.

\section{EXPERIMENTAL-BASED ASSESSMENT}

The main purpose of our experimental analysis is to compare the neural techniques introduced in Sect. III across the datasets described in Section IV. Aimed at a fair comparison, we adopt a 10-fold cross validation for each experiment. The model structure for each algorithm and the pertinent hyperparameters are summarized in Table III.

The whole assessment comprises: 


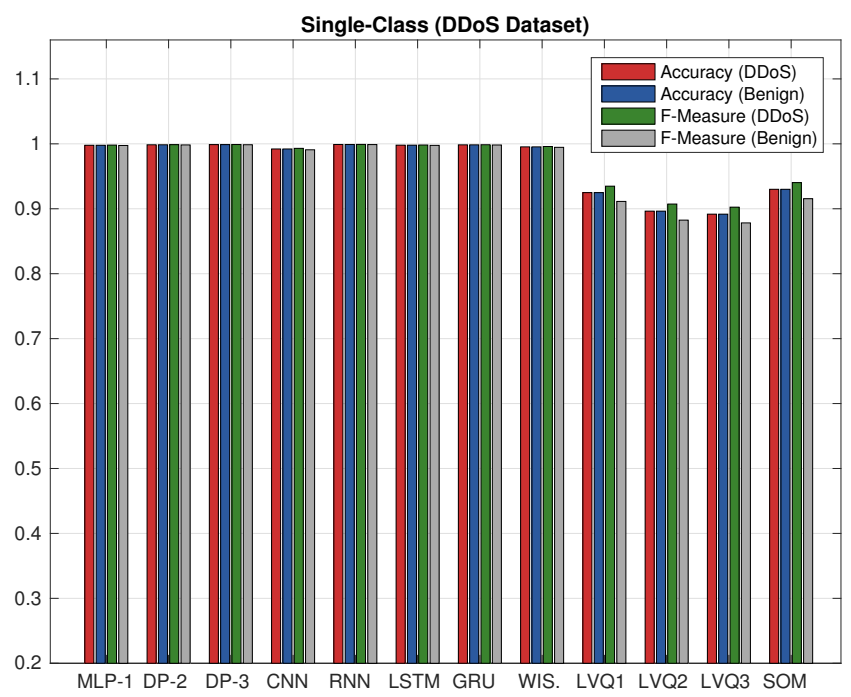

(a)

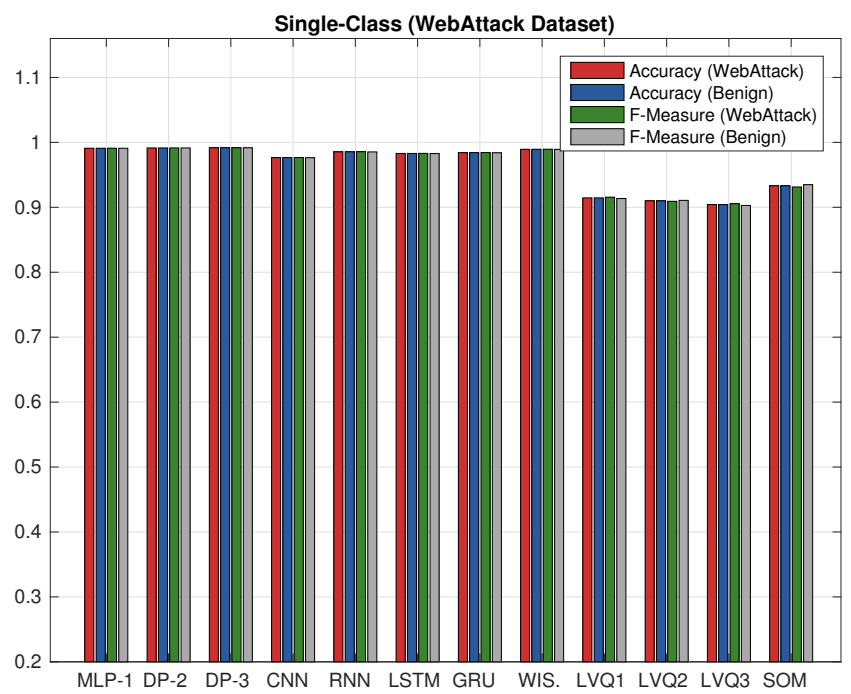

(c)

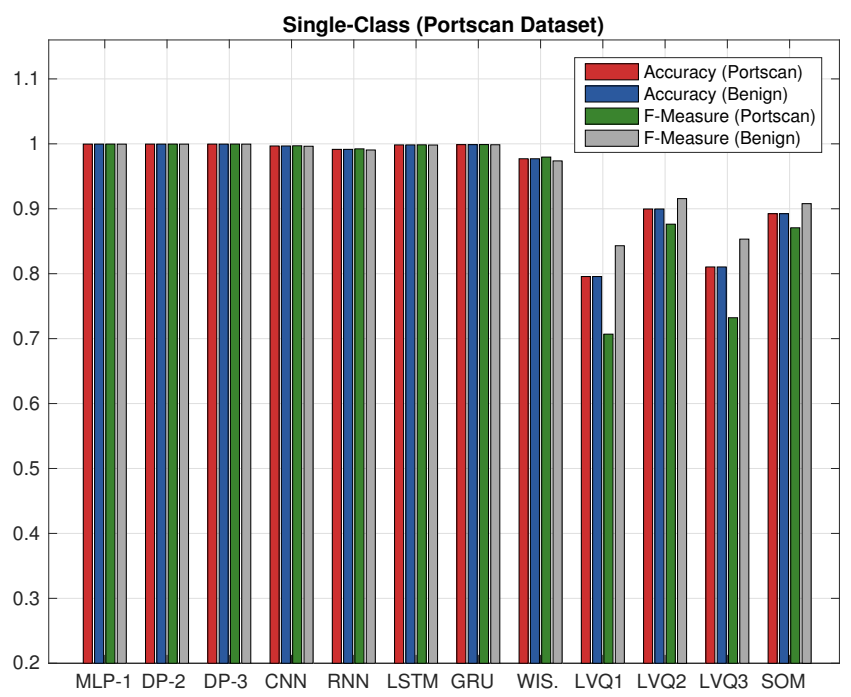

(b)

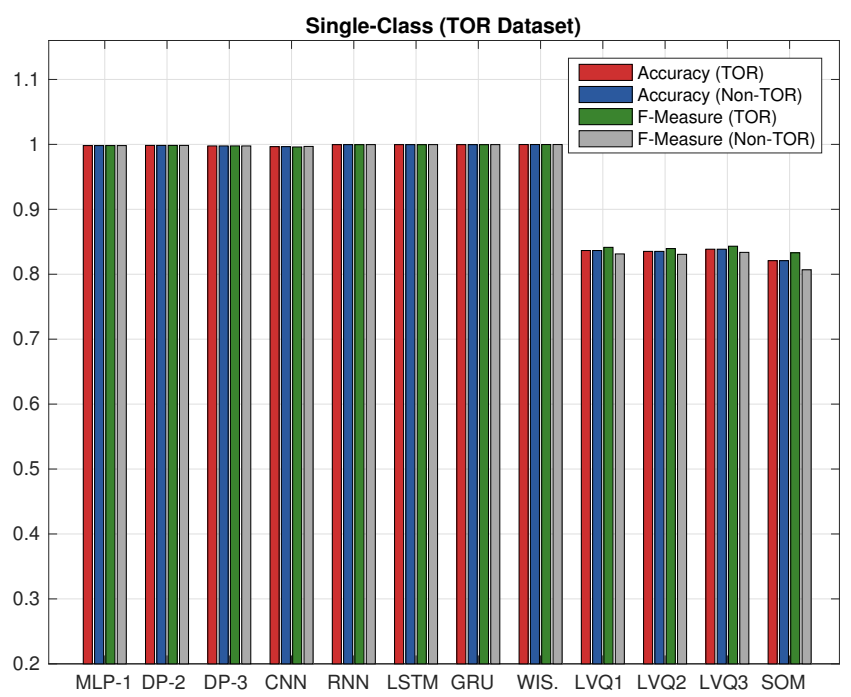

(d)

Fig. 3: Performance in terms of Accuracy/F-Measure for different single-class datasets: (a) DDoS; (b) Portscan, (c) WebAttack, (d) TOR.

- Performance analysis: obtained by evaluating two metrics typically used in the field of traffic classification [88], [89], viz.

- Accuracy : ratio of correctly predicted observations to the total, calculated by

$$
\frac{T P+T N}{T P+T N+F P+F N} ;
$$

- F-Measure : an indicator of a per-class performance, calculated by

$$
2 \cdot \frac{\text { Precision } \cdot \text { Recall }}{\text { Precision }+ \text { Recall }},
$$

where Precision is the ratio of correctly classified traffic over the total predicted traffic in a class, and Recall is the ratio of correctly classified traffic over all ground truth traffic in a class.
- Time complexity analysis: derived by measuring the whole classification process (including training time) as the number of instances grows from $10^{3}$ to $2 \cdot 10^{4}$.

We perform the overall analysis on a PC equipped with Intel CoreTM i5-7200U CPU@ 2.50GHz CPU and 16 GB of RAM. For the sake of convenience, we split our analysis in two: the single-class and the multi-class cases.

\section{A. Single-Class Analysis}

We start by evaluating the performance of the different neural-based techniques by considering four single-class datasets (DDoS, Portscan, WebAttack, and TOR), reporting accuracy and F-measure in Figures 3 (a), 3(b), 3(c), and 3 (d), respectively. The choice of four completely different datasets (representative of four substantially different network attacks) is useful to verify the effectiveness of the tested algorithms and their relationships with the data. 


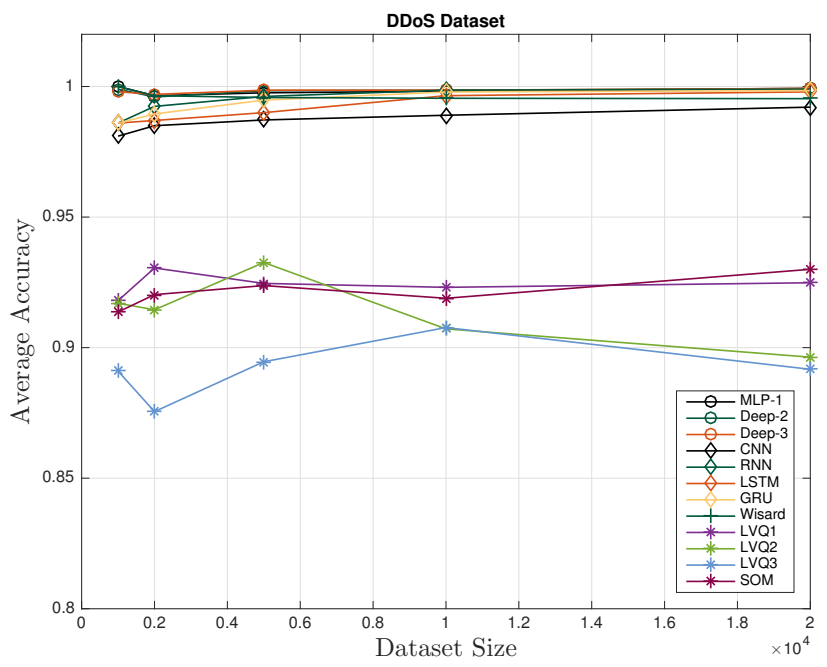

(a)

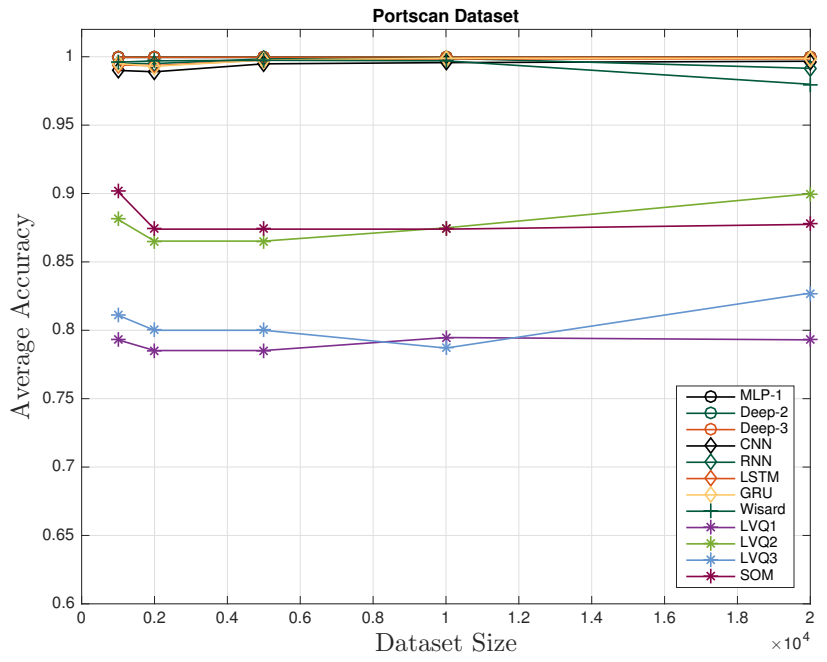

(c)

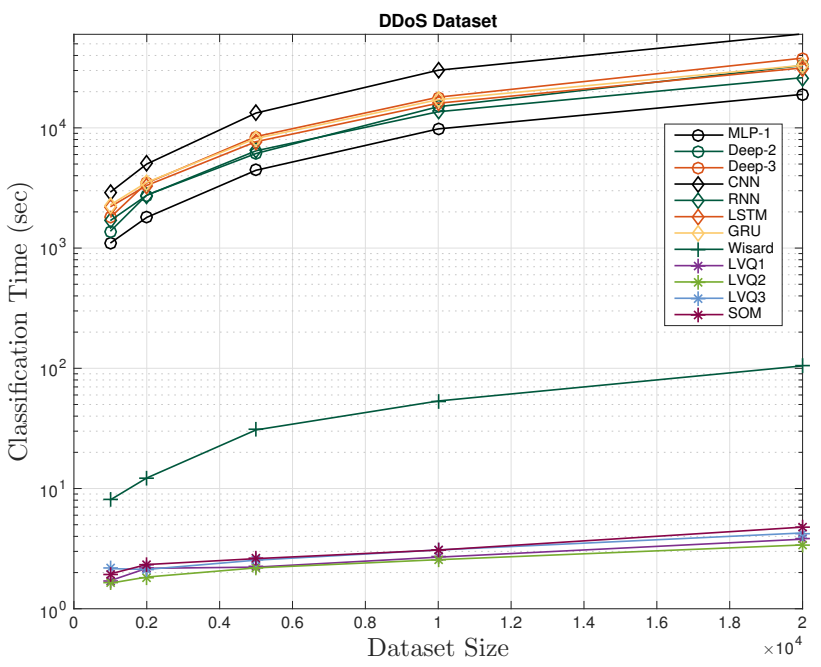

(b)

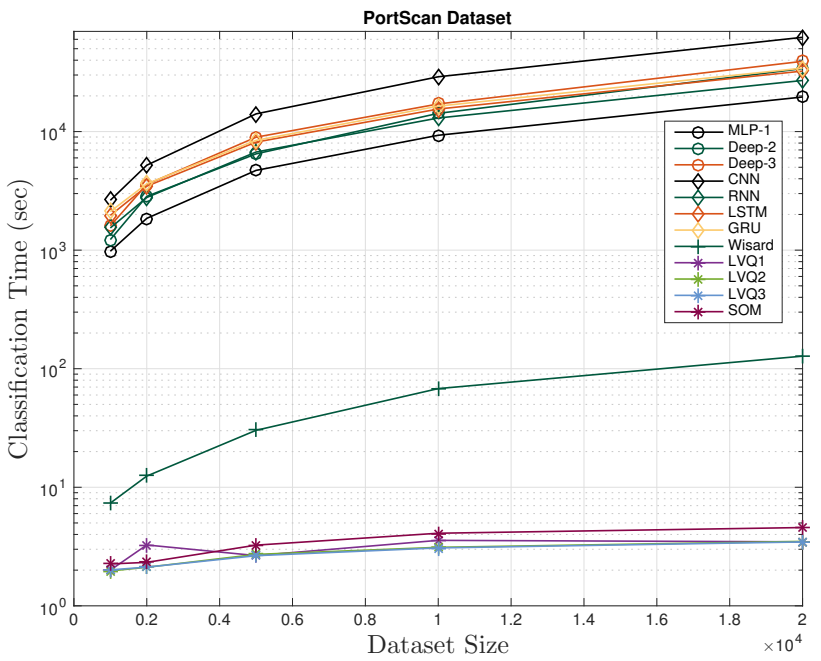

(d)

Fig. 4: Single-class (DDoS): (a) Average Accuracy vs. dataset size; (b) Classification Time vs. dataset size; Single-class (Portscan): (c) Average Accuracy vs. dataset size; (d) Classification Time vs. dataset size.

Among classic ANN-based algorithms we distinguish between deep versions (Deep-2, Deep-3) and non-deep ones (MLP-1) whose detailed structure is reported in Table III] MLP-1 refers to a standard MLP with 3 layers: 1 input layer, 1 hidden layer, and 1 output layer. On the other hand, deep versions are indicated by Deep-2 (1 input layer, 2 hidden layer, 1 output layer) and Deep-3 (1 input layer, 3 hidden layer, 1 output layer). In order to compare algorithms with similar performances, MLP-1, Deep-2 and Deep-3 have been set with the same number of weights $(2 k)$. This is about one order of magnitude smaller than the dataset size, according to what is recommended in the literature [90], [91]. We also note that the number of weights for MLP-1, Deep-2 and Deep3 directly results from the different number of neurons chosen per each hidden layer, namely: 26 neurons for MLP-1, 23 and 10 neurons for the two hidden layers of Deep-2, and 20, 16, and 11 neurons for the 3 hidden layers of Deep-3 (see also Table III). As concerns the evolved deep models (CNN, RNN,
LSTM, GRU), details about their structures are available in Table III Also for these approaches, the choice of a particular structure (e.g. the number of filters in $\mathrm{CNN}$, the number of recurrent units in RNN, and so forth), is aimed at obtaining a number of weights comparable with MLP-1, Deep-2, and Deep-3 so to have a fair comparison.

In our analysis, the MLP-based versions (MLP-1, Deep-2 and Deep-3) and the evolved deep techniques (CNN, RNN, LSTM, GRU) exhibit good values of average accuracy (all around 0.99) for all the four examined datasets (panels of Fig. 3). Similarly, for all the aforementioned algorithms and for each dataset, F-measure exhibits high values, indicating a very negligible fraction of false positives and false negatives. Similar high performance is exhibited by Wisard for all the datasets with a slight exception of Portscan Dataset. By contrast, LVQs and SOM methodologies have lower performance and seem to suffer from the presence of more false positives and false negatives. This is visible for the Portscan dataset (Fig. 3(b)) 
that exhibits an oscillating F-measure, which can be ascribed to the different codebook vectors obtained when considering different input datasets. In particular, competitive learning methods seem suffer from the particular type of attack dataset. In fact, the Euclidean distance (which is used as the main metric within the discussed competitive learning approaches) may not perfectly capture the properties of various datasets, being they structurally different. This is due to the fact that Euclidean distance is a not scale invariant measure, thus, it has difficulty to deal with vector components having different dynamic range. This is also the reason why the 3 variants of LVQ can behave slightly differently when considering diverse datasets.

Another useful analysis is aimed at evaluating the impact of dataset size on performance. For the sake of compactness, we choose DDoS and Portscan as benchmark datasets, being DDoS and Portscan representative of two completely different and well structured network attacks. Precisely, we evaluate the average accuracy (between DDoS/Portscan and benign classes) against an interval of dataset size varying between $10^{3}$ and $2 \cdot 10^{4}$ instances. The results shown in Figures 4 a) and 4 (c) provide a clear indication that: $i$ ) both ANNs and WiSARD exhibit very stable performance as dataset size varies; ii) LVQ shows some minor fluctuations, with an average accuracy that is just slightly under 0.9 in few cases. In this case, fluctuations can be ascribed to the codebook size that would require heuristical adjustments as the dataset size varies.

For the sake of fairness, the performance comparison must be complemented by a time-complexity analysis, as shown in Figs. 4(b-d). Therein, classification time (in seconds) is plotted against the number of instances (from $10^{3}$ to $2 \cdot 10^{4}$ ). For both experiments (DDoS dataset in 4(b) and Portscan dataset in 4 (d)) we can reasonably recognize three slots in the $\mathrm{Y}$ axis: the first one, ranging from $10^{3}$ to $15 \cdot 10^{4}$ seconds, where MLP-1, Deep-2, 3 and evolved deep architectures (CNN, RNN, LSTM, GRU) operate (slowest algorithms); the second one, ranging from about 10 to $10^{2}$ seconds which includes WiSARD (medium-fast algorithm); the third one, ranging from about 1 to 5 seconds, including LVQ1, LVQ2, LVQ3, and SOM (faster algorithms).

We are not surprised that deep networks are up to 2 orders of magnitude slower w.r.t. other techniques, due to the fully connected neurons between each layer. As regards the evolved deep architectures, time complexity is typically associated to the adoption of more sophisticated structures such as the convolutional layers (CNNs), or mechanisms to retain the internal memory states (RNN, LSTM, GRU).

On the other hand, approaches based on competitive learning (LVQs, SOM) are faster since only the winner neurons are enabled to update their weights, although this comes at the cost of a lower accuracy. Surprisingly, the best trade-off between accuracy and time complexity is offered by WiSARD. Its RAM-based structure, in fact, allows a fast learning stage since it deals with binary information regardless on data dimension. In terms of time complexity, the feed-forward incremental learning scheme implemented in WiSARD is more advantageous than classic backpropagation, requiring many iterations to converge.

\section{B. Multi-Class Analysis}

Multi-class analysis is useful to analyze how the various algorithms react when dealing with different classes of traffic. With this aim, we built a 4-class dataset by mixing (in a balanced way) three malicious classes (DDoS, Portscan, and Adware) with a Benign class. The rationale behind this choice is to take into account a mix of peculiar threats. As regards the performance analysis, for the sake of readiness, we show multi-class accuracy and F-measure separately in Figs. 5(a) and 5(b), respectively. As a general trend, we observe a satisfactory classification performance by MLP and Deep algorithms. WiSARD exhibits good performance in classifying DDoS and Portscan (0.9957 and 0.9952 accuracy, respectively), but is poor in classifying Adware and Benign classes (0.784 and 0.782 accuracy, respectively). The reason is to be found behind the structural difference between those different types of attacks. DDoS and Portscan are both highly "structured" attacks. The former can be characterized in terms of high rate of messages that a bot sends to a victim; Portscan hides continuous ping-based requests towards a target in order to unveil possible open ports. By contrast, Adwares can be easily confused within a benign flow, since they just convey annoying banners, as often occurs also within legitimate web portals. The situation changes drastically when we look at the remaining algorithms (LVQs and SOM), where false positives have high weight especially for Portscan, Adware, and Benign classes, as shown in Fig. 5(b). Also, the F-measure is often below 0.4 in case of Adware and Benign classes. With respect to the single-class case, here, the effects produced by the codebook class assignment and peculiarity of Euclidean measure are amplified.

Finally, among competitive-based approaches, LVQ-3 exhibits the best performance, due to the introduction of the empirical parameter $\epsilon$ (see Sect. III-D3), which helps to better regulating the distance between the data and the pertinent codebook vectors. This notwithstanding, all the considered techniques exhibit fairly stable accuracy averages, across the whole range of dataset sizes (from $10^{3}$ to $2 \cdot 10^{4}$ instances), as revealed by Fig. 6(a). As expected, the average accuracy in all cases pertaining the multi-class experiment is slightly lower than their single-class counterpart, due to the fact that classifying more than two classes is a more challenging task.

Let us now discover if the multi-class analysis has an impact on the time complexity of the considered algorithms. Figure 6(b) shows how the order of magnitude in classification times per algorithm is similar w.r.t. the case of single-class analysis. As to be expected, the slight increase in classification time going from single to multi-class cases can be ascribed to the higher number of different traffic flows to be classified. For example, along the varying dataset size $\left[10^{3}, 2 \cdot 10^{3}, 5 \cdot 10^{3}, 10^{4}, 2 \cdot 10^{4}\right]$, WiSARD exhibits the following (approximated) classification times: $[8.047,12.24,30.731,53.474,104.783]$ seconds in the single class case, and $[8.389,15.058,31.831,61.958,119.848]$ seconds in the multi-class case. Once again, WiSARD appears to provide the optimal trade-off between performance needs and time complexity issues. 


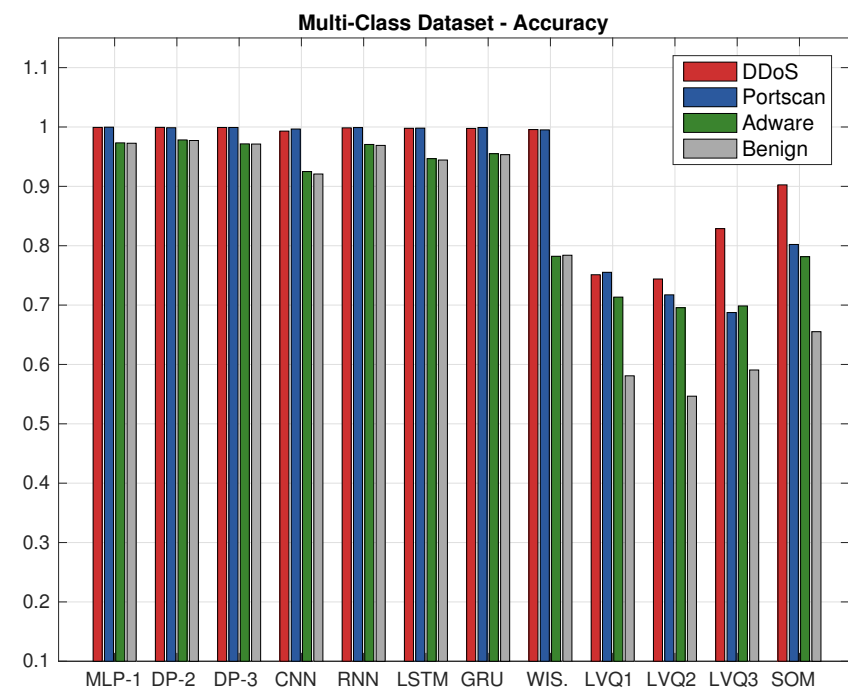

(a)

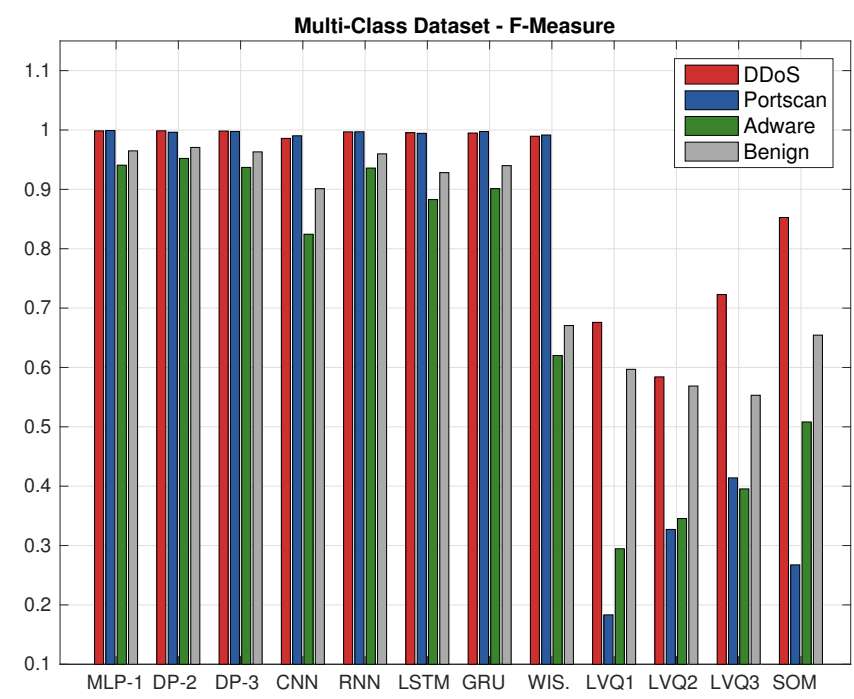

(b)

Fig. 5: Performance analysis for a 4-classes dataset: (a) Accuracy, (b) F-Measure.

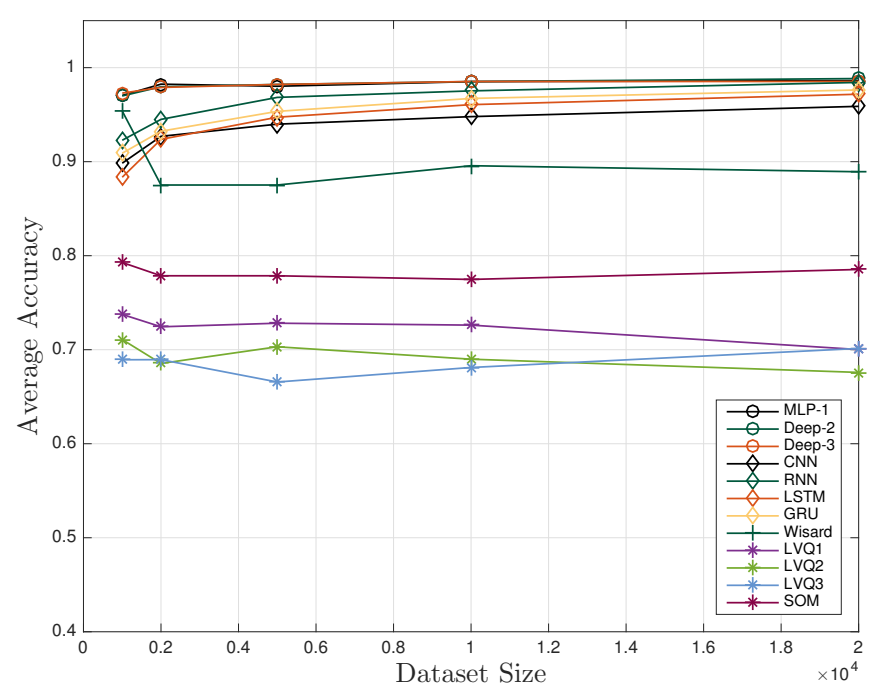

(a)

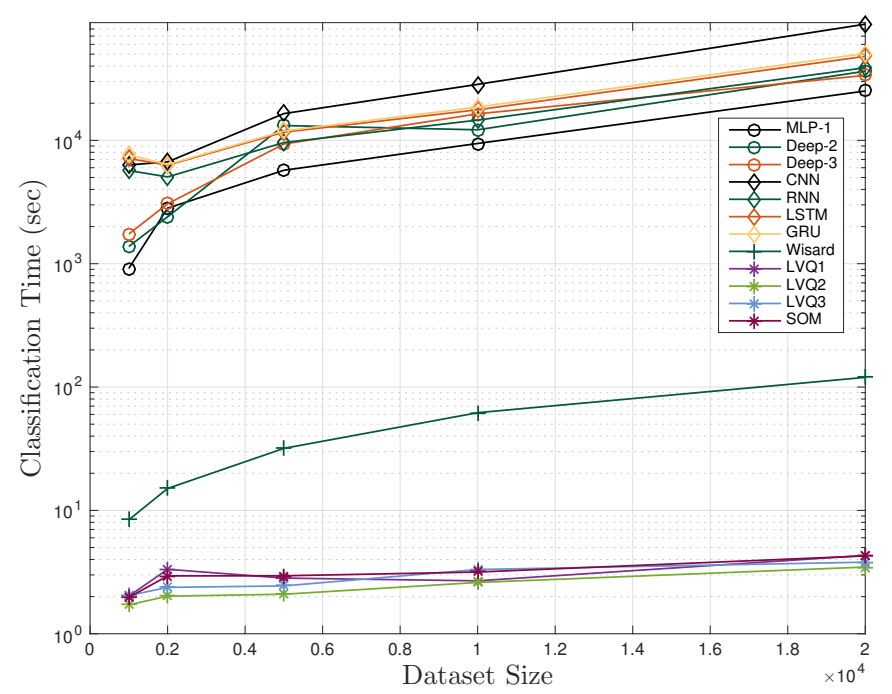

(b)

Fig. 6: Multi-class dataset: (a) Average Accuracy vs. dataset size;

(b) Classification Time vs. dataset size.

\section{General Considerations}

A number of interesting considerations may be derived from our comparative analysis. First, not all neural-based techniques are equally applicable to network intrusion detection, particularly when performance is the key issue.

ANN approaches (including modern deep-based methods) tend to offer noteworthy accuracy, both in single-class and multi-class datasets. Unfortunately, though, accuracy comes at the expenses of time complexity, which will demand for deepbased techniques to rely on specialized GPU-based hardware.

Moreover, as an auxiliary investigation pertaining the deep approach, we evaluate the impact of the updating weight mechanism on the overall performance, expressed through the Mean Square Error (MSE). The behavior is shown in
Figs. 7(a) and 7(b) for the single and multi-class datasets, respectively, evaluated over 100 epochs. Such analysis reveals that most techniques exhibit limited fluctuations around the median value of MSE and a quite low MSE value. The only exception is given by CNN where the higher MSE is reasonably due to the complexity of the convolution operation. Similar considerations raised in a bio-informatic study [92] where only MLP and CNN techniques have been compared. Detailed values are reported in Table IV, in terms of median and inter-quartile range (difference between third and first quartile) values. On average, the most "stable" techniques are the classic Deep-2 and Deep-3 architectures. The addition of a bit more sophisticated structure (e.g. convolutional layer for CNN, LSTM/GRU units, and so forth) can translate into MSE 


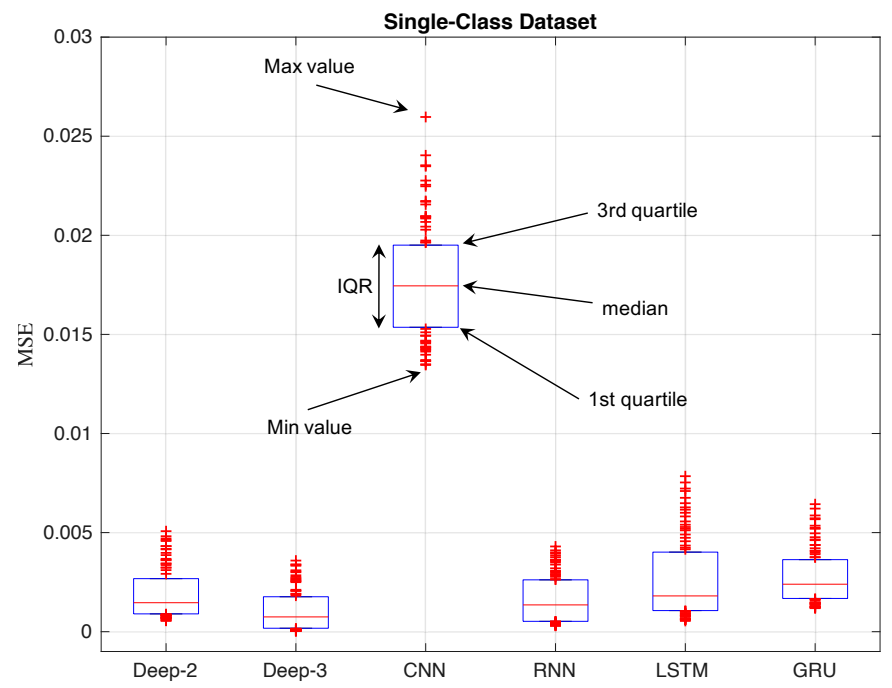

(a)

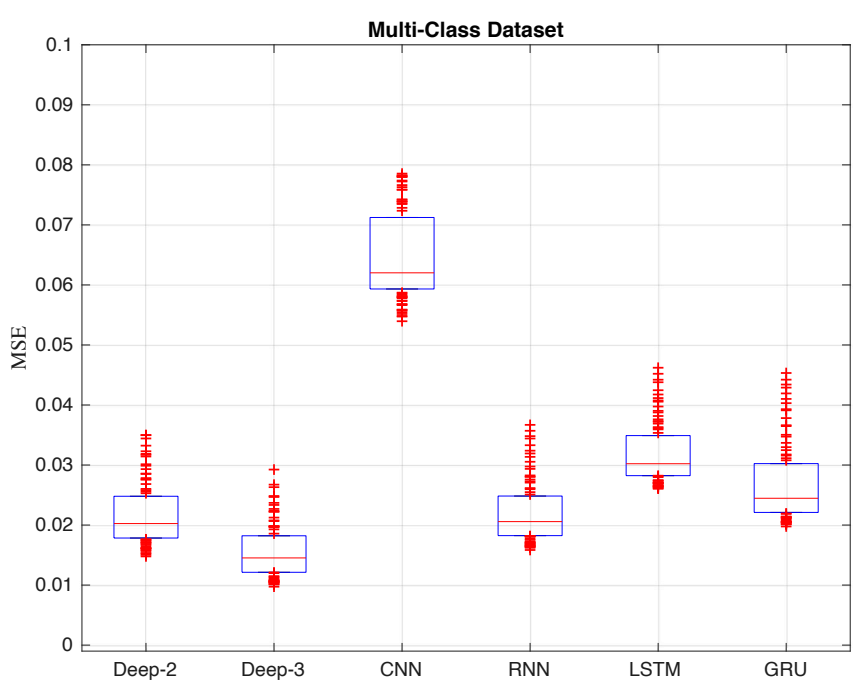

(b)

Fig. 7: MSE analysis when introducing more sophisticated deep models (CNN, RNN, LSTM, GRU). Single-class (DDoS benchmark dataset) (a), Multi-class (DDoS-Portscan-Adware-Benign dataset) (b).

fluctuations due to the presence of additional hyperparameters to tune. Obviously, such fluctuations directly reflect the internal structure of each deep technique and can be more (e.g. $\mathrm{CNN}$ ) or less (e.g. RNN) pronounced.

TABLE IV: Median and IQR values (MSE analysis) for deepbased techniques

\begin{tabular}{ccccc} 
& \multicolumn{2}{c}{ Median } & \multicolumn{2}{c}{ IQR } \\
\hline Technique & Single-Class & Multi-Class & Single-Class & Multi-Class \\
\hline Deep-2 & $1.5 \cdot 10^{-3}$ & $2.02 \cdot 10^{-2}$ & $1.8 \cdot 10^{-3}$ & $7 \cdot 10^{-3}$ \\
Deep-3 & $7.4 \cdot 10^{-4}$ & $1.45 \cdot 10^{-2}$ & $1.6 \cdot 10^{-3}$ & $6.1 \cdot 10^{-3}$ \\
CNN & $1.74 \cdot 10^{-2}$ & $6.2 \cdot 10^{-2}$ & $4.1 \cdot 10^{-3}$ & $1.19 \cdot 10^{-2}$ \\
RNN & $1.4 \cdot 10^{-3}$ & $2.06 \cdot 10^{-2}$ & $2.1 \cdot 10^{-3}$ & $6.6 \cdot 10^{-3}$ \\
LSTM & $1.8 \cdot 10^{-3}$ & $3.02 \cdot 10^{-2}$ & $2.9 \cdot 10^{-3}$ & $6.7 \cdot 10^{-3}$ \\
GRU & $2.4 \cdot 10^{-3}$ & $2.45 \cdot 10^{-2}$ & $2 \cdot 10^{-3}$ & $8.1 \cdot 10^{-3}$ \\
\hline
\end{tabular}

Remarkably, deep-learning techniques could find interesting application in the network intrusion management field when the training set exhibits slow time dynamics. In this case, the training operation (being time/resource consuming) could be performed only once (or performed rarely across the time).

As regards competitive learning techniques (LVQ in the 3 variants and $\mathrm{SOM}$ ), pros and cons are (almost) reversed w.r.t. ANN algorithms. In the case of single-class analysis, a dependency on the type of dataset is highlighted (better performance in DDoS dataset than in Portscan). This is reasonably due to the fact that different inputs produce different mappings with codebook vectors. As to be expected, this effect is further amplified in multi-class datasets, where false positives and false negatives lead to unstable f-measure figures (Fig. 5 (b)).

However, the competitive learning algorithms exhibit very appealing time complexity figures (up to 3 orders of magnitude lower than ANNs), thanks to their approach to taking into account only the "winner" neurons during the whole classification process. Accordingly, cooperative techniques could find useful application in highly time-variant intrusion detection settings, where it is crucial to quickly adapt to the dataset variations. Possibly, they can operate an early (even rough) detection to be refined later by means of other algorithms.

Finally, an unexpected finding is offered by WiSARD that, through its weightless mechanism, provides the best tradeoff between performance (accuracy/F-measure) and time complexity, in both single and multi-class cases. This outstanding behavior is mainly due to two aspects: first, the binarization scheme (jointly with the RAM-like neurons structure) adopted by WiSARD allows to handle multivariable data in a fast way. Then, the training stage follows an incremental approach since each sample reinforces the past knowledge in updating the network state; this implies a fast convergence and makes WiSARD particularly suitable in domains where online learning is crucial. What is even more interesting is that WiSARD has hardly ever been considered in the field of traffic flow classification, while it appears to be a very interesting candidate to be exploited in intrusion detection. This WNN-based approach should certainly be considered as a promising alternative to existing methods, particularly for its potential to meet the near-real-time constraints involved in NIDS classification problems.

\section{CONCLUSION}

This work explores the applicability of prominent neuralbased techniques in network intrusion detection. We carry out an experiment-based comparison to quantify performance and trade-off achievable with each solution. Our aim to perform a fair comparison has directed our investigation to focus on artificial neural networks (ANN). This was to avoid the typical issues arising when comparing ML methods relying on different rationale (e.g. ANN vs. SVM vs. Decision Trees).

In the related work section, we have provided pointers to numerous other surveys, illustrating similarities (in aims) and 
differences (in methodologies). The key peculiarity of our paper is its experimental-based approach to reviewing alternative ANN options. We based our evaluation on modern datasets (CIC-IDS-2017/2018), while most of the earlier experimental results are based on the outdated KDD99 dataset (reflecting network attack issues that have largely been solved). Our review provides useful performance (in terms of accuracy and F-measure) and time complexity data, providing the basis for a trade-off analysis across different ANNs such as deep networks or competitive learning-based networks. To add value to our study, we wanted to consider also methods that have not typically been employed in intrusion detection, particularly the weightless neural networks (WNNs).

The outcomes reveal a number of interesting findings. i) ANN-based approaches (including deep networks) are characterized by outstanding performance in almost all cases (as to be expected). Yet, they suffer the drawback of being slow due to the underlying backpropagation algorithm, which is typically slow to converge. ii) Neural-based techniques relying on a competitive learning approach (LVQ1, LVQ2, LVQ3, SOM) overturn this perspective, thanks to a much reduced time complexity (due to the winner-neuron mechanism). However, this comes with a lower performance when different datasets are considered (due to the different mapping mechanism between input and codebook vectors). iii) The WiSARD algorithm (representative of WNNs) exhibits a surprisingly best tradeoff in terms of performance and time complexity, making it an appealing candidate to operate in conjunction with intrusion detection systems.

The proposed analysis could be extended in several ways. The first (and perhaps natural) direction is to better investigate the potential of weightless neural networks in the context of network intrusion detection. Beyond WiSARD, in fact, other WNN algorithms such as Probabilistic Logic Node (PLN), Goal Seeking Neuron (GSN), or General Neural Unit (GNU) could reveal noteworthy properties when applied in this field. Then, we would suggest to explore how the addition of a feature selection (FS) pre-processing stage would reduce time complexity. This could be particularly advantageous for deep techniques (the most critical in terms of time constraints), even if the adoption of a non well designed FS strategy could result in a further computational overhead. Finally, having identified pros and cons of neural-based techniques in the field of intrusion detection, an engine which allows to automatically select (or combine) the NN-based strategies best fitting the underlying network environment (e.g. hugely vs. scarcely time-variant traffic profiles) can be designed. This latter point could have intriguing implications onto prospective $6 G$ scenarios which, according to the network experts, will be characterized by automatic service provisioning, intelligent resource management, and smart network adjustments.

\section{REFERENCES}

[1] I. Possebon, A. Santos da Silva, L. Zambenedetti Granville, A SchaefferFilho, and A.K. Marnerides. Improved network traffic classification using ensemble learning. In 2019 IEEE Symposium on Computers and Communications, pages 1-6, 2019.
[2] F. Grando, L. Zambenedetti Granville, and L.C. Lamb. Machine learning in network centrality measures: Tutorial and outlook. ACM Comput. Surv., 51(5):102:1-102:32, 2018.

[3] Cisco Encrypted Traffic Analysis. https://www.cisco.com/c/dam/en/ us/solutions/collateral/enterprise-networks/enterprise-network-security/ nb-09-encrytd-traf-anlytcs-wp-cte-en.pdf Accessed: 2020-02-25.

[4] L. P. Gaspary, E. Meneghetti, and L. R. Tarouco. An SNMP agent for stateful intrusion inspection. In IFIP/IEEE Eighth International Symposium on Integrated Network Management, 2003., pages 3-16, 2003.

[5] L. P. Gaspary, R. N. Sanchez, D. W. Antunes, and E. Meneghetti. A SNMP -based platform for distributed stateful intrusion detection in enterprise networks. IEEE Journal on Selected Areas in Communications, 23(10):1973-1982, 2005.

[6] W. Cerroni, G. Moro, R. Pasolini, and M. Ramilli. Decentralized detection of network attacks through $\mathrm{p} 2 \mathrm{p}$ data clustering of SNMP data. Computers \& Security, 52:1 - 16, 2015.

[7] W. Cerroni, G. Moro, R. Pasolini, and M. Ramilli. Network attack detection based on peer-to-peer clustering of SNMP data. In Lecture Notes of the Institute for Computer Sciences, volume 22, 2009.

[8] W. Cerroni, G. Moro, T. Pirini, and M. Ramilli. Peer-to-peer data mining classifiers for decentralized detection of network attacks. In Proceedings of the Twenty-Fourth Australasian Database Conference - Volume 137, pages 101-107, 2013.

[9] Kdd cup 1999 data. http://kdd.ics.uci.edu/databases/kddcup99/ kddcup99.html Accessed: 2020-02-25.

[10] M. Tavallaee, E. Bagheri, W. Lu, and A. A. Ghorbani. A detailed analysis of the KDD CUP 99 data set. In 2009 IEEE Symposium on Computational Intelligence for Security and Defense Applications, pages 1-6, 2009.

[11] Sharafaldin I., Habibi Lashkari A., and Ghorbani A.A. Toward generating a new intrusion detection dataset and intrusion traffic characterization. In 4th International Conference on Information Systems Security and Privacy, 2018.

[12] M. Di Mauro and C. Di Sarno. Improving siem capabilities through an enhanced probe for encrypted Skype traffic detection. Journal of Information Security and Applications, 38:85-95, 2018.

[13] F. Cauteruccio, G. Fortino, A. Guerrieri, A. Liotta, D.C. Mocanu, C. Perra, G. Terracina, and M. Torres Vega. Short-long term anomaly detection in wireless sensor networks based on machine learning and multi-parameterized edit distance. Information Fusion, 52:13 - 30, 2019.

[14] I. Hafeez, M. Antikainen, A. Y. Ding, and S. Tarkoma. IoT-keeper: Detecting malicious IoT network activity using online traffic analysis at the edge. IEEE Transactions on Network and Service Management, pages 1-1, 2020.

[15] M. Casillo, S. Coppola, M. De Santo, F. Pascale, and E Santonicola Embedded intrusion detection system for detecting attacks over canbus. In 4th International Conference on System Reliability and Safety, pages 136-141, 2019.

[16] R. U. Khan, X. Zhang, M. Alazab, and R. Kumar. An improved convolutional neural network model for intrusion detection in networks. In 2019 Cybersecurity and Cyberforensics Conference, pages 74-77, 2019.

[17] S. T. F. Al-Janabi and H. A. Saeed. A neural network based anomaly intrusion detection system. In Developments in E-systems Engineering, pages 221-226, 2011.

[18] K. A. Taher, B. Mohammed Yasin Jisan, and M. M. Rahman. Network intrusion detection using supervised machine learning technique with feature selection. In 2019 International Conference on Robotics,Electrical and Signal Processing Techniques (ICREST), pages 643-646, 2019.

[19] S. Kumar and A. Yadav. Increasing performance of intrusion detection system using neural network. In IEEE International Conference on Advanced Communications, Control and Computing Technologies, pages 546-550, 2014

[20] D. Papamartzivanos, F. Gomez Marmol, and G. Kambourakis. Introducing deep learning self-adaptive misuse network intrusion detection systems. IEEE Access, 7:13546-13560, 2019.

[21] Z. T. Fernando, I. S. Thaseen, and C. A. Kumar. Network attacks identification using consistency based feature selection and self organizing maps. In First International Conference on Networks Soft Computing, pages 162-166, 2014.

[22] S. McElwee and J. Cannady. Improving the performance of selforganizing maps for intrusion detection. In SoutheastCon 2016, pages $1-6,2016$.

[23] C. Li-ying, Z. Xiao-xian, L. He, and C. Gui-fen. A network intrusion detection method based on combined model. In International Conference 
on Mechatronic Science, Electric Engineering and Computer, pages 254-257, 2011.

[24] Z.N.Al-Sultani R.S.Naoum. Learning vector quantization (lvq) and knearest neighbor for intrusion classification. World of Computer Science and Information Technology Journal, 2(3):105-109, 2012.

[25] J. Woo, J. Song, and Y. Choi. Performance enhancement of deep neural network using feature selection and preprocessing for intrusion detection. In 2019 International Conference on Artificial Intelligence in Information and Communication (ICAIIC), pages 415-417, 2019.

[26] Y. Jia, M. Wang, and Y. Wang. Network intrusion detection algorithm based on deep neural network. IET Information Security, 13(1):48-53, 2019.

[27] A. Nagisetty and G. P. Gupta. Framework for detection of malicious activities in IoT networks using Keras deep learning library. In 2019 3rd International Conference on Computing Methodologies and Communication (ICCMC), pages 633-637, 2019.

[28] F. A. Khan, A. Gumaei, A. Derhab, and A. Hussain. A novel two-stage deep learning model for efficient network intrusion detection. IEEE Access, 7:30373-30385, 2019.

[29] Z. Zhang and P. Pan. A hybrid intrusion detection method based on improved fuzzy c-means and support vector machine. In 2019 International Conference on Communications, Information System and Computer Engineering (CISCE), pages 210-214, 2019.

[30] W. Wang, X. Du, and N. Wang. Building a cloud ids using an efficient feature selection method and svm. IEEE Access, 7:1345-1354, 2019.

[31] X. Tang, S. X. . Tan, and H. Chen. SVM based intrusion detection using nonlinear scaling scheme. In 2018 14th IEEE International Conference on Solid-State and Integrated Circuit Technology (ICSICT), pages 1-4, 2018.

[32] S. Sun, Z. Ye, L. Yan, J. Su, and R. Wang. Wrapper feature selection based on lightning attachment procedure optimization and support vector machine for intrusion detection. In (IDAACS-SWS), pages 41-46, 2018.

[33] S. Teng, N. Wu, H. Zhu, L. Teng, and W. Zhang. Svm-dt-based adaptive and collaborative intrusion detection. IEEE/CAA Journal of Automatica Sinica, 5(1):108-118, 2018.

[34] A. Hadri, K. Chougdali, and R. Touahni. A network intrusion detection based on improved nonlinear fuzzy robust PCA. In 2018 IEEE 5th International Congress on Information Science and Technology (CiSt), pages $636-641,2018$

[35] F. Meng, Y. Fu, F. Lou, and Z. Chen. An effective network attack detection method based on kernel PCA and LSTM-RNN. In 2017 International Conference on Computer Systems, Electronics and Control (ICCSEC), pages 568-572, 2017.

[36] K. Ibrahimi and M. Ouaddane. Management of intrusion detection systems based-KDD99: Analysis with LDA and PCA. In 2017 International Conference on Wireless Networks and Mobile Communications (WINCOM), pages 1-6, 2017.

[37] S. M. Almansob and S. S. Lomte. Addressing challenges for intrusion detection system using naive Bayes and PCA algorithm. In 2017 2nd International Conference for Convergence in Technology (I2CT), pages $565-568,2017$.

[38] A. A. Aburomman and M. B. I. Reaz. Ensemble of binary SVM classifiers based on PCA and LDA feature extraction for intrusion detection. In IEEE (IMCEC), pages 636-640, 2016.

[39] R. Primartha and B. A. Tama. Anomaly detection using random forest: A performance revisited. In 2017 International Conference on Data and Software Engineering (ICoDSE), pages 1-6, 2017.

[40] X. Gao, C. Shan, C. Hu, Z. Niu, and Z. Liu. An adaptive ensemble machine learning model for intrusion detection. IEEE Access, 7:8251282521, 2019.

[41] N. Kumar, H. Akash, R. A. Prataap, G. Srinath, and C. Mala. Intelligent intrusion detection system using decision tree classifier and bootstrap aggregation. In ISED, pages 199-203, 2018.

[42] M. Bitaab and S. Hashemi. Hybrid intrusion detection: Combining decision tree and gaussian mixture model. In 2017 14th International ISC (Iranian Society of Cryptology) Conference on Information Security and Cryptology (ISCISC), pages 8-12, 2017.

[43] M. A. Jabbar and S. Samreen. Intelligent network intrusion detection using alternating decision trees. In 2016 International Conference on Circuits, Controls, Communications and Computing (I4C), pages 1-6, 2016.

[44] Z. Rustam and A. S. Talita. Fuzzy kernel robust clustering for anomaly based intrusion detection. In 2018 Third International Conference on Informatics and Computing (ICIC), pages 1-4, 2018.

[45] K. Alrawashdeh and C. Purdy. Fast hardware assisted online learning using unsupervised deep learning structure for anomaly detection. In
2018 International Conference on Information and Computer Technologies (ICICT), pages 128-134, 2018.

[46] M. Z. Alom and T. M. Taha. Network intrusion detection for cyber security using unsupervised deep learning approaches. In 2017 IEEE National Aerospace and Electronics Conference (NAECON), pages 6369, 2017.

[47] W. Chen, F. Kong, F. Mei, G. Yuan, and B. Li. A novel unsupervised anomaly detection approach for intrusion detection system. In $3 r d$ International conference on big data security on cloud, pages 69-73, 2017.

[48] S. Seo, S. Park, and J. Kim. Improvement of network intrusion detection accuracy by using restricted Boltzmann machine. In 2016 8th International Conference on Computational Intelligence and Communication Networks (CICN), pages 413-417, 2016.

[49] F. Amiri, M. Rezaei Yousefi, C. Lucas, A. Shakery, and N. Yazdani. Cluster ensemble with link-based approach for botnet detection. Journal of Network and Systems Management, 26(3):616 - 639, 2018.

[50] N. Moustafa and J. Slay. UNSW-NB15: a comprehensive data set for network intrusion detection systems (UNSW-NB15 network data set). In 2015 Military Communications and Information Systems Conference (MilCIS), pages 1-6, 2015.

[51] N.Koroniotis, N.Moustafa, E. Sitnikova, and B.Turnbull. Towards the development of realistic botnet dataset in the Internet of Things for network forensic analytics: BoT-IoT dataset. Future Generation Computer Systems, 100:779-796, 2019.

[52] Canadian institute for cybersecurity. https://www.unb.ca/cic/ Accessed: 2020-02-25.

[53] A realistic cyber defense dataset. https://registry.opendata.aws/ cse-cic-ids2018/ Accessed: 2020-02-25

[54] V. Kanimozhi and T. P. Jacob. Artificial intelligence based network intrusion detection with hyper-parameter optimization tuning on the realistic cyber dataset cse-cic-ids2018 using cloud computing. In 2019 International Conference on Communication and Signal Processing, pages 33-36, 2019.

[55] C. Ma, X. Du, and L. Cao. Analysis of multi-types of flow features based on hybrid neural network for improving network anomaly detection. IEEE Access, 7:148363-148380, 2019.

[56] S. Wankhede and D. Kshirsagar. DoS attack detection using machine learning and neural network. In Fourth International Conference on Computing Communication Control and Automation, pages 1-5, 2018.

[57] J. Lee, J. Kim, I. Kim, and K. Han. Cyber threat detection based on artificial neural networks using event profiles. IEEE Access, 7:165607165626, 2019.

[58] T. T. T. Nguyen and G. Armitage. A survey of techniques for internet traffic classification using machine learning. IEEE Communications Surveys Tutorials, 10(4):56-76, 2008.

[59] R. Boutaba, M. A. Salahuddin, N. Limam, S. Ayoubi, N. Shahriar, F. Estrada-Solano, and O. M. Caicedo. A comprehensive survey on machine learning for networking: evolution, applications and research opportunities. Journal of Internet Services and Applications, 9(1):16, 2018.

[60] H. Hindy, D. Brosset, E. Bayne, A. Seeam, C. Tachtatzis, R. Atkinson, and X. Bellekens. A taxonomy and survey of intrusion detection system design techniques, network threats and datasets. CoRR, abs/1806.03517, 2018.

[61] A. Khraisat, I. Gondal, P. Vamplew, and J. Kamruzzaman. Survey of intrusion detection systems: techniques, datasets and challenges. Cybersecurity, 2(1):20, 2019.

[62] A. Aldweesh, A. Derhab, and A. Emam. Deep learning approaches for anomaly-based intrusion detection systems: A survey, taxonomy, and open issues. Knowledge-based Systems, 189:105-124, 2020.

[63] G. Fernandes, J. J. P. C. Rodrigues, L. F. Carvalho, J. F. Al-Muhtadi, and M. L. Proena. A comprehensive survey on network anomaly detection. Telecommunication Systems, 70(3):447-489, 2019.

[64] A. L. Buczak and E. Guven. A survey of data mining and machine learning methods for cyber security intrusion detection. IEEE Соттиnications Surveys Tutorials, 18(2):1153-1176, 2016.

[65] L. N. Tidjon, M. Frappier, and A. Mammar. Intrusion detection systems: A cross-domain overview. IEEE Communications Surveys Tutorials, 21(4):3639-3681, 2019.

[66] H. Azwar, M. Murtaz, M. Siddique, and S. Rehman. Intrusion detection in secure network for cybersecurity systems using machine learning and data mining. In 2018 IEEE 5th International Conference on Engineering Technologies and Applied Sciences (ICETAS), pages 1-9, 2018.

[67] N. Moustafa, J. Hu, and J. Slay. A holistic review of network anomaly detection systems: A comprehensive survey. Journal of Network and Computer Applications, 128:33-55, 2019. 
[68] G. Meena and R. R. Choudhary. A review paper on IDS classification using KDD99 and NSL-KDD dataset in Weka. In 2017 International Conference on Computer, Communications and Electronics (Comptelix), pages 553-558, 2017

[69] R. Ravipati and A. Munther. A survey on different machine learning algorithms and weak classifiers based on KDD and NSL-KDD datasets. International Journal of Artificial Intelligence \& Applications, 10:01-11, 2019.

[70] C. Yin, Y. Zhu, J. Fei, and X. He. A deep learning approach for intrusion detection using recurrent neural networks. IEEE Access, 5:21954-21961, 2017.

[71] H. Bengio. Learning deep architectures for AI. Found. Trends Mach. Learn., 2(1):1-127, 2009.

[72] X. Glorot, A. Bordes, and Y. Bengio. Deep sparse rectifier neural networks. In Proceedings of the Fourteenth International Conference on Artificial Intelligence and Statistics, pages 315-323, 2011.

[73] G. E. Dahl, D. Yu, L. Deng, and A. Acero. Context-dependent pre-trained deep neural networks for large-vocabulary speech recognition. IEEE Transactions on Audio, Speech, and Language Processing, 20(1):30-42, 2012.

[74] M. Ravanelli, P. Brakel, M. Omologo, and Y. Bengio. Light gated recurrent units for speech recognition. IEEE Transactions on Emerging Topics in Computational Intelligence, 2(2):92-102, 2018.

[75] H. Le, I. Oparin, A. Allauzen, J. Gauvain, and F. Yvon. Structured output layer neural network language models for speech recognition. IEEE Transactions on Audio, Speech, and Language Processing, 21(1):197206, 2013.

[76] Y. Lecun and Y. Bengio. Convolutional networks for images, speech and time series. The MIT Press, pages 255-258, 1995.

[77] D.E. Rumelhart, G.E. Hinton, and R.J. Williams. Learning representations by back-propagating errors. Nature, 323(6088):533-536, 1986.

[78] S. Hochreiter and J. Schmidhuber. Long Short-Term Memory. Neural Computation, 9(8):1735-1780, 1997.

[79] K. Cho, B. van Merrienboer, C.. Gülçehre, D. Bahdanau, F. Bougares, H. Schwenk, and Y. Bengio. Learning phrase representations using RNN encoder-decoder for statistical machine translation. In Proceedings of the 2014 Conference on Empirical Methods in Natural Language Processing, pages 1724-1734, 2014.

[80] P. Bowden I. Alexander, W. Thomas. Learning deep architectures for ai. Sensor Review, 4(3):120-124, 1984.

[81] M. De Gregorio and M. Giordano. An experimental evaluation of weightless neural networks for multi-class classification. Applied Soft Computing, 72:338 - 354, 2018.

[82] T. Kohonen. Learning vector quantization. In The Handbook of Brain Theory and Neural Networks (Arbib ed.), pages 631-635, 2003.

[83] T. Kohonen. Self-Organizing Maps, 3rd ed. Springer-Verlag, Berlin, Heidelberg, 2001.

[84] C. C. Aggarwal. Neural Networks and Deep Learning. Springer-Verlag, Gewerbestrasse 11, 6330 Cham, Switzerland, 2018.

[85] D.P. Kingma and L.J. Ba. Adam: A method for stochastic optimization. In ICLR2015, 2015.

[86] V. Matta, M. Di Mauro, and M. Longo. Botnet identification in randomized DDoS attacks. In Proceedings of the 24th European Signal Processing Conference, pages 2260-2264, 2016.

[87] M. E. Ahmed, S. Ullah, and H. Kim. Statistical application fingerprinting for DDoS attack mitigation. IEEE Transactions on Information Forensics and Security, 14(6):1471-1484, 2019.

[88] J. Zhang, Y. Xiang, Y. Wang, W. Zhou, Y. Xiang, and Y. Guan. Network traffic classification using correlation information. IEEE Transactions on Parallel and Distributed Systems, 24(1):104-117, 2013.

[89] A. Alsirhani, S. Sampalli, and P. Bodorik. Ddos detection system: Using a set of classification algorithms controlled by fuzzy logic system in Apache Spark. IEEE Transactions on Network and Service Management, 16(3):936-949, 2019.
[90] P. S. Szczepaniak P. J.G. Lisboa, E. C. Ifeachor. Artificial Neural Networks in Biomedicine. Springer Science, London, 2000.

[91] V. Prasad and S.D. Gupta. Applications And Potentials Of Artificial Neural Networks In Plant Tissue Culture. Springer, Dordrecht, 2008.

[92] S.J. Kim, C. Wang, B. Zhao, H. Im, J. Min, H.J. Choi, J. Hee, J. Tadros, N.T. Choi, C.M. Castro, R. Weissleder, H. Lee, and K. Lee. Deep transfer learning-based hologram classification for molecular diagnostics. Scientific Reports, 8:01-12, 2018.

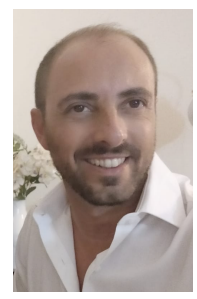

Mario Di Mauro received the Laurea degree in electronic engineering from the University of Salerno (Italy) in 2005, the M.S. degree in networking from the University of L'Aquila (Italy) jointly with the Telecom Italia Centre in 2006, and the PhD. degree in information engineering in 2018 from University of Salerno. He was a Research Engineer with CoRiTel (Research Consortium on Telecommunications, led by Ericsson Lab, Italy) and then a Research Fellow with University of Salerno. He has authored several scientific papers, and holds a patent on a telecommunication aid for impaired people. His main fields of interest include: network security, performance and availability, data analysis, ML techniques.

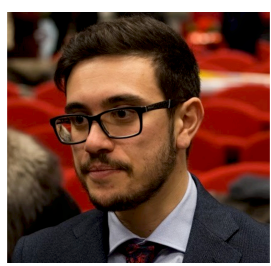

Giovanni Galatro received the Laurea degree (summa cum laude) in information engineering from the University of Salerno (Italy) in 2018, and has been a visiting student at Dept. of Computer Science at Groningen University (Netherlands). In 2017 he got a scholarship with Telecommunication and Applied Statistics groups, focused on the availability analysis of modern telecommunication systems. His main fileds of interest include: network availability and machine learning.

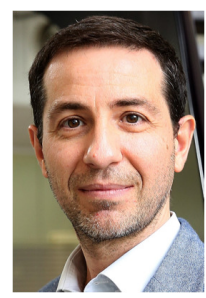

Antonio Liotta (SMEEE15) is Full Professor at the Faculty of Computer Science, Free University of Bozen-Bolzano (Italy), where he teaches Data Science and Computer Networks. Previously, he was full professor at Eindhoven University of Technology (NL), University of Derby (UK), and Edinburgh Napier University (UK). He has also held academic positions at University of Surrey (UK) and Essex University (UK), in addition to Visiting and Distinguished Professorships in the UK, Australia and China. His team is at the forefront of influential research in data science and artificial intelligence, specifically in the contex of smart cities, Internet of Things, and smart sensing. He is renowned for his contributions to miniaturized machine learning, particularly in the context of the Internet of Things. He has led the international team that has recently made a breakthrough in artificial neural networks, using network science to accelerate the training process. Antonio is a Fellow of the U.K. Higher Education Academy. He has 6 patents and over 350 publications to his credit, and is the Editor-in-Chief of the Springer Internet of Things book series. 